\title{
Energy dependent structure of Xe ion tracks in YBCO and the effect on the superconductive properties in magnetic fields
}

Elena I. Suvorova ${ }^{1 *}$, Pavel N. Degtyarenko ${ }^{2,3}$, Igor A. Karateev ${ }^{4}$, Alexey V. Ovcharov ${ }^{4}$, Alexander L. Vasiliev ${ }^{1,4,5}$ Vladimir A. Skuratov ${ }^{6,7,8}$, Philippe A. Buffat ${ }^{9}$

${ }^{1}$ A.V. Shubnikov Institute of Crystallography, Federal Scientific Research Centre "Crystallography and Photonics" of Russian Academy of Sciences, Leninsky pr., 59, 19333 Moscow, Russia

${ }^{2}$ Joint Institute for High Temperatures of Russian Academy of Sciences, Izhorskaya st. 13 Bd.2, 125412, Moscow, Russia

${ }^{3}$ SuperOx, Nauchnyi pr., 20-2, 117246 Moscow, Russia

${ }^{4}$ National Research Center "Kurchatov Institute”, Akademika Kurchatova pl., 1, 123182 Moscow, Russia

${ }^{5}$ Moscow Institute of Physics and Technology (State University), MIPT, 9 Institutskiy per.,

Dolgoprudny, 141701 Moscow Region, Russia

${ }^{6}$ FLNR, JINR, Joliot-Curie St 6, 141980 Dubna, Russia

${ }^{7}$ National Research Nuclear University MEPhI, Kashirskoe shosse, 31, 115409 Moscow, Russia

${ }^{8}$ Dubna State University, 141982, 141982 Dubna, Moscow Region, Russia

${ }^{9}$ Ecole Polytechnique Fédérale de Lausanne, Centre Interdisciplinaire de Microscopie Electronique, MXC, Station 12, CH-1015 Lausanne, Switzerland

* - Corresponding author: suvorova@crys.ras.ru

Current address: A.V. Shubnikov Institute of Crystallography of Russian Academy of Sciences, Leninsky pr., 59, Moscow 119333, Russia.

E-mails:

P. Degtyarenko: degtyarenkopn@gmail.com

I. Karateev: iaKarateev@gmail.com

A. Ovcharov: ovcharov.91@gmail.com

A. Vasiliev: a.vasiliev56@gmail.com

V.Skuratov: skuratov@jinr.ru

P. Buffat: philippe.buffat@epfl.ch 


\begin{abstract}
The morphology and structure of damaged regions (tracks) produced by swift heavy 167, 77 and $46 \mathrm{MeV}^{132} \mathrm{Xe}^{23+}$ ions in YBCO-based second generation industrial high temperature superconductors wires (2G HTS) were studied using transmission electron microscopy. It was shown that ion produce tracks aligned along the ion trajectory but of morphology depending on their energy: continuous, nearly continuous or discontinuous tracks like prolate ellipsoids $10-15 \mathrm{~nm}$ in length and spheroids $5 \mathrm{~nm}$ in diameter. The damaged regions of about $5 \mathrm{~nm}$ in diameter contain an amorphous material with a lower density compared to pristine YBCO. The material density drops from $6300 \mathrm{~kg} \cdot \mathrm{m}^{-3}$ for YBCO matrix to $3600 \mathrm{~kg} \cdot \mathrm{m}^{-3}$ inside the tracks. Barium enrichment was found in the vicinity of the track at a distance of about $10 \mathrm{~nm}$ from the center of the defect. Ion damage tracks with different morphology showed different effectiveness as pinning centers. Tracks composed of discontinuous pinning centers - spheroids of about $5 \mathrm{~nm}$ in diameter - bring the best enhancement of the critical current density. It occurs for the lowest ion energy $(46 \mathrm{MeV})$ in the range of energy loss $8.9 \mathrm{keV} / \mathrm{nm}-4.7 \mathrm{keV} / \mathrm{nm}$ for Xe ions. The samples showed highest critical current density of $56 \mathrm{MA} / \mathrm{cm}^{2}(4.2 \mathrm{~K})$ and $3 \mathrm{MA} / \mathrm{cm}^{2}(77 \mathrm{~K})$ in self-field while in magnetic fields of $8 \mathrm{~T}$ the values of $17 \mathrm{MA} / \mathrm{cm}^{2}(4.2 \mathrm{~K})$ and $1.6 \mathrm{MA} / \mathrm{cm}^{2}(77 \mathrm{~K})$ were achieved. The reduction of the superconducting volume corresponding to the amorphous radiation defects did not exceed $4 \%$ from the total sample volume.
\end{abstract}

Keywords: 2G HTS coated conductors, YBCO, ion irradiation damage, structure, critical current density, transmission electron microscopy. 


\section{INTRODUCTION}

We report on the study of chemical composition, density, morphology and nanostructure down to the atomic level of damaged regions (tracks) in $\mathrm{YBa}_{2} \mathrm{Cu}_{3} \mathrm{O}_{7-\delta}(\delta \leq 0.1)$ created with the $\mathrm{Xe}$ ions with energy below $1 \mathrm{MeV} /$ nucleon using transmission electron microscopy (TEM), electron energy loss spectroscopy (EELS) and energy dispersive X-ray spectrometry (EDXS). Our experiments also aimed to find some correlation between morphology of tracks and their efficiency as flux pinning centers to improve conducting properties of $2 \mathrm{G}$ HTS wires in magnetic fields.

The amorphous structure of tracks created by high-energy Xe ions in polycrystalline sintered YBCO samples or thin epitaxial films was described using HRTEM in the first papers by H. Watanabe et al. [1] and D. Bourgault et al. [2], with the volume fraction of amorphous material depending on used fluences [1]. The volume expansion associated with the amorphization causes strains in the surrounding matrix [3]. Observations of a change in TEM contrast allowed Bursill and Braunshausen [4] to conclude that the density within the amorphous tracks made by $14 \mathrm{MeV} /$ nucleon $\mathrm{Pb}$ ions in zircon decreases. As well molecular dynamics simulations and radial distribution functions calculations showed a decrease of the track core density in $\mathrm{BaTiO}_{3}$ after irradiation with $635 \mathrm{MeV}^{238} \mathrm{U}^{+}[5]$.

However, for YBCO, experimental data on the density and composition of ion tracks are still missing. We consider that it is important to get the knowledge and to improve understanding of the material properties modified by heavy ion irradiation. Thanks to the research of Kwon et al. [6], it is known that the amount of oxygen and copper is decreased at the interface between the irradiation track and the YBCO matrix over a length of $\sim 3 \mathrm{~nm}$. Our previous TEM study [7] showed that $107 \mathrm{MeV} \mathrm{Kr}$ ions left damaged regions having a shape close to spherical with a significant decrease in the amount of material up to the void. It is necessary to understand the fate of Ba, which is the heaviest element in YBCO and determines its density.

As known, the morphology of the tracks is strongly dependent on the electronic energy loss $d E / d x$ influenced by several parameters, including the mass and the energy of the irradiating ion. 
Tombrello [8] collected the data indicating that ion tracks in YBCO tend to be continuous for $d E / d x \geq 30 \mathrm{keV} / \mathrm{nm}$, for $20 \leq d E / d x \leq 30 \mathrm{keV} / \mathrm{nm}$ they are nearly continuous with small gaps between sections and for $10 \leq d E / d x \leq 20 \mathrm{keV} / \mathrm{nm}$ the damaged regions are isolated and extend along the ion's path in the form of prolate ellipsoids or spheres below $10 \mathrm{keV} / \mathrm{nm}$.

Marwick et al. [9] claimed that if the length of defects is large, the pinning force should be large. The effectiveness of point defects and small aggregates of impurity atoms or vacancies in pinning the flux lines is not high in HTS cuprates with the short superconducting coherence length $\xi$ and important thermal fluctuations. The strong pinning of flux lines by the columnar damage should occur if the flux lines lie in the damage columns for much of their length. However, it was not specified whether these columns are continuous or discontinuous. The ion energy range used in this work implies the formation of continuous and discontinuous tracks in YBCO. Weinstein et al. [10, 11] gleaned evidences supporting a view about the advantages of discontinuous tracks as pinning centers due to less damage and the important contribution to the entanglement of fluxoids. All their results showed a five times higher critical current density $J_{c}$ than can be achieved with continuous columnar PCs.

Leroux et al. [12] and Rupich et al [13] have demonstrate that 3.5-MeV oxygen and 18-MeV Au ions irradiation with the optimal dose markedly enhances the in-field critical current of commercial 2G HTS tapes. The main scientific target in these works was the creation of a mixed pinning landscape composed of a large number of relatively small irradiation-induced defects coexisting with preexisting defects (oxide precipitates, twin boundaries, point defects).

Furthermore, quantifying the local density of pinning sites utilizing the Voronoi diagram showed the advantages of a random pinscape over ordered arrays of defects in a wide magnetic field range [14]. It is worth to note that this study opens a cheaper road to the irradiation pining approach for production large length $2 \mathrm{G}$ wire using a roll-to-roll irradiation process by using relatively low ion energies (under $20 \mathrm{MeV}$ ) with electrostatic generators available on the market. However, the nature 
and structure of radiation defects created by ions, as well as the state of the crystal structure of the YBCO layer after irradiation were not considered.

It was shown that ion tracks produced by $\mathrm{Xe}$ and $\mathrm{Kr}$ ions with energy in the range between $46 \mathrm{MeV}$ and $167 \mathrm{MeV}$ in a $1-\mu \mathrm{m}$ YBCO film and in $\mathrm{Y}_{2} \mathrm{O}_{3}$ nanoprecipitates were detected when the specific ionization energy loss $d E / d x$ is $4.7 \mathrm{keV} / \mathrm{nm}$ or higher [15]. The value of $4.7 \mathrm{keV} / \mathrm{nm}$ is the stopping power threshold for the formation of amorphous tracks in $\mathrm{YBCO}$, which is almost three times less than the value presented by Zhu et al. in [16].

The irradiation induced damaged regions in YBCO play an important role in the improvement of the superconductivity properties under magnetic fields. The most important barrier to obtain the highest critical density current $J_{c}$ values is in the behavior of vortex matter - vortex lines in superconductor (YBCO in this study) appeared in relatively high magnetic fields. Vortices moving under the electric current applied dissipate energy what resulted in a large loss of current carrying capabilities of the superconductor.

In an effort to shed light on atomic-level processes occurring during the formation of ion tracks of different morphology, the present work combines TEM study of the YBCO microstructure and measurements of the conductive properties of $2 \mathrm{G}$ HTS wires in magnetic fields. The TEM methods were used to determine the change in chemical composition, structure and density of damaged regions in YBCO layers in $2 \mathrm{G}$ HTS wires irradiated with 167, 77 and $46 \mathrm{MeV}{ }^{132} \mathrm{Xe}$ ions (from 0.13 to $0.76 \mathrm{MeV} /$ nucleon). We analyze and compare the relative importance of the morphology of tracks on conductive properties in magnetic field up to $8 \mathrm{~T}$ at different temperatures from 4.2 to $77 \mathrm{~K}$ to support the idea of higher efficiency of discontinuous tracks to increase the critical current density.

\section{METHODS AND MATERIALS}

The 2G HTS coated conductor wires (Super Power Inc.) used in this work have a layered structure made of an YBCO layer of $1 \mu \mathrm{m}$ thick grown on a multilayer buffer $\left(\mathrm{LaMnO}_{3} / \mathrm{MgO} / \mathrm{Y}_{2} \mathrm{O}_{3}\right.$ / $\mathrm{Al}_{2} \mathrm{O}_{3}$ ) on a $100 \mu \mathrm{m}$ thick Hastelloy substrate and with a $2 \mu \mathrm{m}$ of silver cap on its other face. 
Several specimens were cut from the wire into pieces $1.0 \times 0.5 \mathrm{~cm}^{2}$ prior to irradiation. Then specimens were mounted on copper water-cooled holders $\left(20^{\circ} \mathrm{C}\right)$ and were uniformly irradiated at room temperature by scanning the ion beam over their whole surface at an average ion flux of $2 \times 10^{8} \mathrm{~cm}^{-2} \mathrm{~s}^{-1}$ at the IC-100 FLNR JINR cyclotron facility. Irradiation time and absorbers of suitable thicknesses inserted between the Xe ion source and the sample were used to select the ion fluence and energy for sample irradiation: $167 \mathrm{MeV}$ (fluence of $1 \times 10^{11} \mathrm{~cm}^{-2}$ ), 77 and $46 \mathrm{MeV}$ (fluence of $5 \times 10^{11} \mathrm{~cm}^{-2}$ for these two latter).

The electronic $\left(S_{e}\right)$ stopping power of Xe ions, range and changes in the ion energies as they pass through the successive layers in the conductor wire were calculated using the SRIM2013 (Stopping and Range of Ions in Matter) code [17].

Figure 1 sketches the layered structure of the coated conductor sample used in this study, the direction of the Xe ion irradiation with the primary energy values and values reduced after ion passing the Ag coating. For simplicity we will use 167, 77 and $46 \mathrm{MeV}$ to denote the energy of Xe ions, however, we have to keep in mind that the actual ion energy has been reduced before proceeding in the YBCO layer after passing through the $2 \mu \mathrm{m}$ silver cap (see Table 2 below in the text).

The YBCO matrix and ion track morphologies and structures were investigated on TEM samples prepared by focused ion beam cutting (FIB) in plan-view (3 samples in plan-view for each ion energy) and cross-section orientations ( 3 cross-sections for each ion energy) under a FEI Helios Nanolab 600i and a FEI Versa 3D DualBeam. Information was gathered $(200 \mathrm{kV}$ and $80 \mathrm{kV}$ accelerating voltage) under a field emission Tecnai Osiris/FEI microscope in conventional Bright (BF), Dark (DF) and Selected Area Electron Diffraction modes in TEM and in High Angle Annular Dark Field (HAADF) modes in Scanning TEM (STEM). Chemical microanalysis was performed by Energy Dispersive X-Ray Spectrometry (EDXS) under a high collection angle (4 diodes/ $0.9 \mathrm{sr}$ ) ChemiSTEM/FEI) detector coupled to an Esprit/Bruker data processing software in a Tecnai Osiris 
(FEI) and electron energy loss spectroscopy (EELS) using a Gatan parallel EEL spectrometer (0.7 эB energy resolution and $0.1 \mathrm{~nm}$ spatial resolution) in a Titan 80-300 (FEI).

The SAED patterns, (S)TEM images were processed using DigitalMicrograph (Gatan) software and interpreted using the JEMS [18] diffraction and image simulation for sake of comparison with the experimental data.

The measurements of the critical current density in applied magnetic field $(1-8 \mathrm{~T})$ and at different temperatures $(4.2,20,35,50,65$ and $77 \mathrm{~K})$ were performed using a Physical Property Measurement System (PPMS, Quantum Design Inc.) of the samples, which were then used to study the microstructures. The magnetic field was applied along the $c$-axis of YBCO (normal to the flat surface of the tape) with a sweep rate of $10 \mathrm{mT} \cdot \mathrm{s}^{-1}$ on samples of $(3.5 \times 3.5 \times 0.14) \mathrm{mm}^{3}$. The critical temperatures $T_{c}$ of the samples after irradiation were estimated from the temperature dependence of the residual magnetic moment when it vanishes.

\section{RESULTS AND DISCUSSIONS}

\section{A. TEM/HRTEM of Xe ion track structures}

Xe ions with the highest energy (167 MeV at the Ag coated surface and $101 \mathrm{MeV}$ at the YBCO surface) created continuous (or nearly continuous) tracks what corresponds to the calculated electronic energy loss of $22 \mathrm{keV} / \mathrm{nm}$. The discontinuous tracks appeared when samples were irradiated with 77 (34.4 MeV at the YBCO surface) MeV Xe ions what corresponds to the calculated electronic energy loss of about $15.0 \mathrm{keV} / \mathrm{nm}$. All these values are below the numbers reported in $[19,20]$ for YBCO. Table 1 shows the actual upper and lower energy and electronic energy loss values calculated using the SRIM2013 code for each primary ion energy and summarized the track morphology characteristic for the specific ion energies. 
Table I. The Xe ion energy, electronic stopping power $d E / d x$, morphology and the specific features of radiation induced ion tracks observed by TEM.

\begin{tabular}{|c|c|c|c|c|c|c|}
\hline \multicolumn{3}{|c|}{ Ion energy $(\mathrm{MeV})$} & \multicolumn{2}{|c|}{$d E / d x(\mathrm{keV} / \mathrm{nm})$} & \multirow{2}{*}{$\begin{array}{c}\text { Track } \\
\text { morphology }\end{array}$} & \multirow[t]{2}{*}{ Track features } \\
\hline $\begin{array}{c}\text { entering } \\
\text { Ag } \\
\text { coating }\end{array}$ & $\begin{array}{c}\text { entering } \\
\text { YBCO }\end{array}$ & $\begin{array}{l}\text { exiting } \\
\text { YBCO }\end{array}$ & $\begin{array}{c}\text { Entrance } \\
\text { YBCO }\end{array}$ & $\begin{array}{c}\text { Exit } \\
\text { YBCO }\end{array}$ & & \\
\hline 167 & 101 & 80.6 & 29.8 & 16.1 & $\begin{array}{l}\text { Continuous } \\
\text { (nearly } \\
\text { continuous) }\end{array}$ & $\begin{array}{l}\text { Diameter of columnar tracks: } 3- \\
5 \mathrm{~nm} \text {, } \\
\text { average distance between tracks: } \\
40 \mathrm{~nm}\end{array}$ \\
\hline 77 & 34.4 & 23.3 & 15.3 & 7.5 & Discontinuous & $\begin{array}{l}\text { Diameter of fragments: } 3-5 \mathrm{~nm} \text {, } \\
5 \mathrm{~nm} \times 5-20 \mathrm{~nm} \text { ellipsoids and } 3 \\
-5 \mathrm{~nm} \text { spheres, } 5-10 \mathrm{~nm} \text { gaps } \\
\text { along tracks } \\
\text { average distance between tracks: } \\
20-25 \mathrm{~nm}\end{array}$ \\
\hline 46 & 17.4 & 11.0 & 8.9 & 4.7 & Discontinuous & $\begin{array}{l}\text { Diameter of fragments: } 3-5 \mathrm{~nm} \text {, } \\
3-5 \mathrm{~nm} \text { spheres, } 5-10 \mathrm{~nm} \text { gaps } \\
\text { along tracks } \\
\text { average distance between tracks: } \\
20-25 \mathrm{~nm}\end{array}$ \\
\hline
\end{tabular}

The contrast of tracks on TEM images strongly depends on the diffraction conditions which are changing because of the foil deformation under the electron beam and grain structure of the sample. While the contrast of tracks on HAADF STEM images depends on chemical composition (average atomic number or the difference in density of track material and the matrix) and in less extent on the diffraction conditions as well. Therefore, the absence of track images does not mean the absence of tracks in the samples, and their visibility is determined by imaging conditions and material properties.

Continuous (or nearly continuous) and discontinuous Xe ion tracks of about $5 \mathrm{~nm}$ wide in YBCO in [100] TEM/HRTEM cross-sections are shown in Figs. 2 - 4. The direction of Xe ions is parallel to the [001] YBCO axis for the presented in Figs. 2 - 4 TEM cross-sections. Continuous tracks of about $5 \mathrm{~nm}$ wide from $167 \mathrm{MeV}$ Xe ions are visible from the Ag cap (black areas at the top of low magnification image in Fig. 2a) to the buffer layers and well aligned parallel to the [001] YBCO axis. 
The tracks formed with the $77 \mathrm{MeV}$ Xe ions contain ellipsoids with the long diameter up to $20-25 \mathrm{~nm}$ and the spheroidal damaged regions with the gaps between defects of about $10 \mathrm{~nm}$ (Fig. $3)$.

Less energetic Xe ions (46 MeV) produce discontinues tracks made up of spheroidal damaged regions ("string-of-beads") with the gaps between spheroids in the range from 3 to $10 \mathrm{~nm}$ along the track direction parallel to the [001] YBCO (Fig. 4). Thus, the long axis of ellipsoids (77 $\mathrm{MeV} \mathrm{Xe}$ ions) decreases with $S_{e}$ (i.e. with decreasing ion energy). The small sizes of the defects created by less energetic ions and the low contrast of the defects make it difficult to observe the radiation tracks and study their morphology.

The amorphous structure of tracks in HRTEM cross sectional images is revealed if the cut of the sample passes through its center (for instance, Fig. 3 c). Otherwise, when the radiation defect is buried in the matrix, the YBCO crystal lattice is visible, and only a change in TEM contrast due to strains in matrix indicates the presence of tracks.

The $d E / d x \approx 4.7 \mathrm{keV} / \mathrm{nm}$ is found to be the threshold value of energy loss at which the radiation damaged regions (tracks) are visible in plan-view samples. The sizes and densities of damaged regions were measured in TEM and STEM plan-views (Fig. 5) using the homemade program AnNa [21]. About 1000 tracks for each ion energy were examined, and their densities of $1 \cdot 10^{11} \mathrm{~cm}^{-2}$ and $4-7 \cdot 10^{11} \mathrm{~cm}^{-2}$ are in good agreement with the ion fluences applied. This means that one track was created by one ion.

The TEM plan-views and the corresponding SAED patterns are shown in Fig. 5. The distances measured in plan-views between tracks are in the range from 10 to $100 \mathrm{~nm}$ and the average spacings are about $40 \mathrm{~nm}$ and $20-25 \mathrm{~nm}$ for the fluencies $1 \cdot 10^{11} \mathrm{~cm}^{-2}$ and $5 \cdot 10^{11} \mathrm{~cm}^{-2}$, respectively. The SAED patterns (Fig. 5 b, f, d) and nanodiffraction (Fig. 5h) clearly show that after irradiation to fluences of $1-5 \cdot 10^{11} \mathrm{~cm}^{-2}$ YBCO keeps the crystalline orthorhombic structure. While electron nanodiffraction patterns obtained from the ion track (Fig. 5i) with probe diameter of about $2 \mathrm{~nm}$ (Fig. 5d) evidenced that material of the track is amorphous. 
If we compare the damaged regions in the Figures $5 \mathrm{a}$ and $5 \mathrm{e}$, the sizes of the defects formed by irradiation with the $46 \mathrm{MeV} \mathrm{Xe}$ ions seems at a first glance smaller than the sizes of defects produced with more energetic $167 \mathrm{MeV}$ Xe ions. Actually, in low or intermediate magnification TEM plan-views we can measure the size of projections of defects buried at different depth of the sample. The variation of the contrast of circular damaged regions in discontinuous tracks $(46 \mathrm{MeV}$ and $77 \mathrm{MeV}$ Xe ions) depends on their depth in the thin TEM sample. The maximum diameter of defect projections with the best contrast can be measured if the sample is cut in the middle of the defect. The true sizes of tracks can be measured in the HRTEM/HRSTEM images (Fig. 6-8). As well, HRTEM study in the present work serves the purpose of clarifying the details of the formation of tracks and understanding whether solidification or (re)crystallization in the track can occur.

Atom lattice has completely disappeared in continuous tracks created with high energetic Xe ions to let a structureless circular area of about $5 \mathrm{~nm}$ in diameter (Fig. 8). However, we can see some lattice fringes in the tracks made with less energetic ions of 77 and $46 \mathrm{MeV}$ (Fig. 6 a and 7 c).

Comparison of experimental HRTEM images obtained from the samples irradiated with ions of different energies with HRTEM image simulations (Figs. 6 - 8) made with the multislice method allows to estimate the TEM sample thickness in the regions of interest. HRTEM simulation of pristine YBCO areas was performed using the YBCO perfect crystal structure [22] and several zone axes [001], [101], [011] and TEM sample thickness up to $40 \mathrm{~nm}$. The microscope parameters were the following: accelerating voltage is $200 \mathrm{kV}$, spherical aberration $\mathrm{C}_{\mathrm{s}}=1.2 \mathrm{~mm}$, beam convergence semi-angle is $0.5 \mathrm{mrad}$, and objective underdefocus is ranging from 0 to $75 \mathrm{~nm}$.

Insets in Figures $6-8$ correspond to the best fits with experimental HRTEM images using the sample thickness as an open parameter in each image and the crystal orientation measured on Fast Fourier Transforms (FFT diffractograms) of HRTEM images. The small difference between the YBCO $\boldsymbol{a}$ and $\boldsymbol{b}$ parameters $(0.3824$ and $0.3888 \mathrm{~nm}$, respectively) requires a precise calibration to differentiate the grain orientations [001] or [011] or [101]. 
The results of HRTEM simulation can be interpreted if we suggest that the radiation defects as fragments of discontinuous tracks in Fig. $6-7$ being spheroids or ellipsoids were not buried completely in the matrix in our thin TEM sample and their circular cross-sections are partly covered with the remaining YBCO crystal giving the lattice fringes contrast. The partially (or completely) covered damaged regions are always slightly brighter compared to the surrounding matrix on HRTEM images. If the track passes through the entire thickness of the TEM sample, as is usually the case for samples irradiated with high-energy ions, although this can occur in samples irradiated with lower-energy ions, then only a structureless region is observed without any lattice fringes contrast on the image (Fig. 8c). The HRTEM imaging coupled with simulation clearly shows that only solidification of the molten material inside the tracks takes place.

\section{B. Chemical composition and density of the material inside the tracks}

\section{EELS}

Electron energy loss spectroscopy (EELS) was applied first to ascertain the local orientation and oxygen content using the $\mathrm{O} 1 \mathrm{~s}(531 \mathrm{eV})$ and $\mathrm{Cu} 2 \mathrm{p} 3 / 2(931-934 \mathrm{eV})$ in the vicinity of tracks in samples irradiated with 46 and $167 \mathrm{MeV}$ Xe ions. Comparison of the experimental EEL spectra with the orientation-dependent EELS measurements for $\mathrm{YBa}_{2} \mathrm{Cu}_{3} \mathrm{O}_{6}$ and $\mathrm{YBa}_{2} \mathrm{Cu}_{3} \mathrm{O}_{7}$ films in [23] led to the conclusion that our results were obtained for YBCO with composition of $\mathrm{YBa}_{2} \mathrm{Cu}_{3} \mathrm{O}_{6.8-6.9}$ in crystal orientation [001].

The EELS drift corrected scans across radiation tracks showed a strong decrease in the Ba content inside the continuous tracks (after irradiation with $167 \mathrm{MeV}$ ions, Fig. 9) and a less significant drop in the damaged regions buried in the sample (mainly after irradiation with $46 \mathrm{MeV}$ ions, Fig. 10). Figure 9a shows a STEM image with a track across which the spectra were obtained at points $1-7$, and a reference area with an yttria precipitate (highlighted in square) relative to which the sample drift was corrected. The diameter of the electron probe was $1 \mathrm{~nm}$. EEL spectra with two Ba-M5,4 edges (804 eV and $788 \mathrm{eV}$, correspondingly) are present in Fig.9 b, c. The lines intensity inside the track significantly decreased compared to the pristine YBCO crystal. A much 
smaller decrease in line intensity in points 2 and 3 compared to 1 and 2 was observed for damaged regions located inside the matrix (Fig. 10).

The EELS measurements did not give an unambiguous answer to the question about the change in the $\mathrm{O}, \mathrm{Cu}$, and $\mathrm{Y}$ content in the tracks, since the intensity fluctuations averaged over several tracks were within the measurement error. Solving this problem requires additional research, including the preparation of samples with graded thicknesses.

There was also no answer to the question of where the barium is localized after leaving the track area. This task was solved using energy dispersive X-ray spectrometry.

\section{EDXS}

Energy dispersive X-ray spectrometry (EDXS) in a transmission electron microscope is the straightest way to get the chemical composition of the materials filling continuous tracks through the TEM sample or around them. For tracks consisting of small ellipsoidal or spheroidal defects, partially or entirely buried in the samples, quantitative analysis would require an accurate measurement of the partition between the defect material and pristine YBCO inside each track.

The simplest way to turn this issue is to focus the attention on tracks where nanodiffraction does not show any remaining traces of crystal structure that allows to consider that the track contains only the defect material. YBCO is sensitive to electron irradiation and care was taken to reduce damage while performing microanalysis with enough statistical relevancy. Therefore, EDXS study was carried out at $80 \mathrm{kV}$ accelerating voltage and the total electron dose was kept below the value of $2 \cdot 10^{-11} \mathrm{Cb} / \mathrm{nm}^{2}\left(\sim 1 \cdot 10^{8}\right.$ electrons $\left./ \mathrm{nm}^{2}\right)$.

Quantitative EDXS analysis was carried out using the Cliff-Lorimer standard-less method with thickness correction on thirty damaged areas. The thickness was estimated to match the $\mathrm{Y}: \mathrm{Ba}: \mathrm{Cu}$ to 1:2:3 in pristine YBCO next to the track. EDX spectra and element maps were acquired for different specimen thicknesses in the range from 10 to $200 \mathrm{~nm}$ and different acquisition times.

Figure 11 shows the HAADF image of tracks (Fig. 11 a) and the corresponding EDXS map (Fig. $11 \mathrm{~b}$ ). Element net counts along pairs of orthogonal line scans are compared in Figures 11.1 to 
11.4 for the two tracks pointed out by arrows. Comparing the net peak intensity (intensity of the characteristic X-ray above background) of damaged areas to that of the YBCO matrix, shows that all element counts are lower in the track areas than in the matrix. However, $\mathrm{Y}$ is reduced to a less extent than $\mathrm{Ba}$ and $\mathrm{Cu}$. The bumps (arrows in Fig. 11.3) on the Ba profile shows that the white contrast around the track core on HAADF image comes from enrichment in the track surroundings within $10 \mathrm{~nm}$ from the center. The trend of intensity change of oxygen is not so clear due to uncertainties on the $\mathrm{O}-\mathrm{K} / \mathrm{Ba}-\mathrm{M}$ deconvolution and Bremsstrahlung background subtraction. Samarium is an impurity present in YBCO (average 1.4 at\%). Red areas around 10nm in diameter are yttria precipitates.

The two defects analyzed in Fig 11 a (arrows) show similar net counts behaviors though their lengths (sample thickness) are quite different. This allows to attribute the lowering of net counts in defects to a change of density at contrary of a surface effect. Assuming that the interatomic distances in the amorphous defect is close to that in the YBCO matrix the density $D_{\text {track }}$ of the defect material can be derived from its atom concentration:

$$
D_{\text {track }}=D_{Y B C O} \cdot W_{\text {track }} / W_{Y B C O}
$$

with $W=N_{Y} \cdot A_{Y}+N_{B a} \cdot A_{B a}+N_{C u} \cdot A_{C u}+N_{O} \cdot A_{O}+N_{S m} \cdot A_{S m}$, where $N$ is the EDXS net counts and $A$ is the atomic weight.

In thick sample part of the electron beam scatters out of the track into pristine YBCO due to i) electron elastic scattering, ii) a possible misorientation between ion and electron beams trajectories. This bias makes the apparent density to reach around $5.6 \mathrm{~g} \mathrm{~cm}^{-3}$ for the worst cases. The most reliable estimate of density in defect $D_{\text {track }}=3600 \mathrm{~kg} \cdot \mathrm{m}^{-3}$ was obtained in thin sample areas and is significantly lower than the YBCO density DҮвСо $=6300 \mathrm{~kg} \cdot \mathrm{m}^{-3}$.

\section{Note on ion track formation mechanism.}

High energy Xe ions of $0.76 \mathrm{MeV} /$ nucleon $(\mathrm{E}=101 \mathrm{MeV})$ in this work formed straight continuous ion tracks throughout the entire $1 \mu \mathrm{m}$ YBCO layer. While at intermediate energies of Xe 
ions of 0.26 and $0.13 \mathrm{MeV} /$ nucleon (34.4 and 17.4 MeV, respectively), discontinuous tracks consisting of defects in the form of elongated ellipsoids and / or spheroids were observed.

According to Komarov et al. [24 - 26] the mechanism of formation of discontinuous tracks should take in account the statistical fluctuations of ion charge caused by the atomic stripping and trapping electrons. Such fluctuations become very important when the ion energy is close to the energy threshold required for a track formation and may change the energy released to the electron subsystem changing continuous tracks in discontinuous ones along certain parts of the ion trajectory. The path length between ion charge changes will determine the length of the defective region in the discontinuous track and the distance between such defects. Therefore, it was argued that the model of charge fluctuations makes it possible to estimate quantitatively the longitudinal size of the defects in discontinuous tracks. However, his comparison with experimental TEM images led him to use additional parameters and coefficients to his approach in order to get a satisfactory agreement with the experimental data.

Tombrello [8] followed a different approach to determine the size of defects in discontinuous track. He assumed that tracks are formed mainly because the ionization of the inner shells what leads to an intensive production of secondary $\delta$-electrons. Again, this requires the introduction of different coefficients proper to the material in order to fit calculations on experimental data. Application of his empirical formulas [8] to the data obtained in our work showed some overestimation of the calculated transverse sizes of damaged regions in the discontinuous tracks. In addition, for more than 200 defects, we did not observe a significant difference in the radii of the tracks formed by $\mathrm{Xe}(0.13,0.26,0.76 \mathrm{MeV} /$ nucleon $)$ ions, which should follow from the formulas.

The mass transport during ion track formation and the reduction of material density inside $\mathrm{Au}$ ion tracks were investigated using small angle x-ray scattering (SAXS) and modeled using molecular dynamics simulations in amorphous and crystalline $\mathrm{SiO}_{2}$ by Klutz et al. [27]. The electronic energy lost by heavy ions was modeled by depositing kinetic energy in random directions 
to the atoms in a $11.5 \times 11.5 \times 5.8 \mathrm{~nm}^{3}$ cell. His simulations and synchrotron SAXS measurements reveal a fine structure of swift heavy ion tracks in $\mathrm{a}-\mathrm{SiO}_{2}$ consisting of a core and a shell of lower and higher densities respectively compared to that one of pristine $\mathrm{SiO}_{2}$.

In our work, the observed tracks which core had density almost half of that of the YBCO matrix surrounded by a shell enriched with Ba. Thus, it can be concluded that such structures are consistent with a frozen-in pressure wave originating from the center of the ion track as a result of the thermal spike. The experimental results obtained in this work show that the mechanism and computational approach of irradiation damage by swift ions still need to be refined and supported by more experimental evidences.

\section{Critical current and pining force measurements in magnetic fields.}

Ion fluencies of $1 \cdot 10^{11}$ and $5 \cdot 10^{11} \mathrm{~cm}^{-2}$ were chosen to create radiation defects playing the role of pinning centers, in addition to epy already existing yttria precipitates. The YBCO microstructure starts to degrade, and the critical current density $J_{c}$ decreases abruptly when higher $\left(\approx 10^{12} \mathrm{~cm}^{-2}\right)$ fluences are applied [7].

Table II shows that $T_{\boldsymbol{c}}$ did not lower dramatically after irradiation (accuracy of temperature measurements was $\pm 0.2 \mathrm{~K})$.

Table II. Transition temperature $T_{c}$ in non- and irradiated samples

\begin{tabular}{cc}
\hline \hline Energy $[\mathrm{MeV}]$ & $\begin{array}{c}\text { Transition temperature, } \\
T_{c}[\mathrm{~K}]\end{array}$ \\
\cline { 2 - 2 } nonirradiated & 90.5 \\
167 & 89.2 \\
77 & 88.9 \\
46 & 88.0 \\
\hline
\end{tabular}

The critical current density $J_{c}$ at temperatures $4.2,20,35,50,65$ and $77 \mathrm{~K}$ of the irradiated samples was determined using the hysteresis loops of magnetization applying the modified Bean 
critical model [28] which for B $/ / c$-axis gives $J_{c}=20 \Delta m / a(1-a / 3 b)$, where $a$ and $b$ are width and length of the rectangular film measured in $\mathrm{cm}(\mathrm{b}>\mathrm{a}), \Delta m\left[\mathrm{emu} / \mathrm{cm}^{3}\right]$ is the difference between magnetic moments $m$ on the descending and ascending field branches of the hysteresis loops [29]. Figure 10 shows the change in critical current density $J_{c}$ at temperatures of $77 \mathrm{~K}$ and $4.2 \mathrm{~K}$ as a function of the magnetic field $B(T)$ for samples irradiated with 46, 77 and $167 \mathrm{MeV}$ Xe ions. As expected, the largest values of the critical current density are obtained at the lowest temperature of 4.2 K for all samples and all applied magnetic fields (Fig. 10) while at high temperatures, a thermally activated process allows part of vortices to depin.

A rather surprising result was the low absolute values of the critical current density especially under the self-field conditions for the sample irradiated with $77 \mathrm{MeV}$ Xe ions (fluence $5 \cdot 10^{11} \mathrm{~cm}^{-2}$ ) even in comparison with the reference sample (Fig. 12 a and c). Nearly no difference in $J_{c}$ values in the fields starting from 3T in samples irradiated with 77 and $167 \mathrm{MeV}$ Xe ions. While the difference in $J_{c}$ values reaches a twofold increase in magnetic fields from 5 to $8 \mathrm{~T}$ in the range of temperatures from $50 \mathrm{~K}$ to $4.2 \mathrm{~K}$ in samples irradiated with ions with the highest $(167 \mathrm{MeV})$ and lowest (46 MeV) energies (Fig. 13) in favor of the latter. This observation suggests that it is not necessary to use high energy ions in order to obtain an enhanced critical current density in moderate magnetic fields.

An increase in critical current density correlates with increasing pinning force (Fig. 14) at low $(4.2-35 \mathrm{~K})$ temperatures in the moderate magnetic fields.

The plots on Figure 15 report the measurements of the so called lift-factor, which is the ratio of the critical current $J_{c}$ at a given temperature and magnetic field to the critical current of the same sample at $77 \mathrm{~K}$ in its self-field $J_{c}$ s.f. Lift-factors made a very clear comparison of the irradiation efficiency by Xe ions of different energies in magnetic fields of 1 and $8 \mathrm{~T}$ and temperatures $77 \mathrm{~K}$ and 4.2 K. The maximum lift-factors can be reached in samples irradiated with the less energetic ions and the greatest difference occurred in $8 \mathrm{~T}$ magnetic field (Fig. $15 \mathrm{~b}$ ). We see a doubling of the lift-factor 
at $4.2 \mathrm{~K}$ for samples irradiated with ions with energies of 46 and $77 \mathrm{MeV}$ and an increase of more than $50 \%$ in samples irradiated with ions with an energy of $167 \mathrm{MeV}$ compared to the pristine wires.

The tendency to increase the lift-factor in the irradiated samples remains at higher temperatures but the difference is significantly reduced. In low fields, for example, $\mathrm{B}=1 \mathrm{~T}$, an increase in the lift-factor in the irradiated samples compared to the pristine ones is significant (Fig. 15 a). However, almost no difference is observed between the values of lift-factors in samples irradiated with high and low energetic ions.

We estimated the damaged fraction of the YBCO material in samples with continuous tracks as tubes of $5 \mathrm{~nm}$ in diameter extending through the whole layer with a surface density of $10^{11} \mathrm{~cm}^{-2}$ and for discontinuous tracks as a raw of spheres of $5 \mathrm{~nm}$ in diameter, $10 \mathrm{~nm}$ long gaps between them and with a surface density of $5 \cdot 10^{11} \mathrm{~cm}^{-2}$. These calculations give approximately $2 \%$ value of damaged volume for the sample with continuous tracks $(167 \mathrm{MeV} \mathrm{Xe})$ and $3.3 \%$ for the sample with discontinues tracks (46 MeV Xe). The fraction values of $2 \%$ and $10 \%$, respectively, one can obtain using the exponential dependence $N=1-e^{-\pi r^{2} \Phi}$, where $N$ is the damaged fraction, $r$ is track radius from TEM observation and $\Phi$ is the ion fluence. It is clear that the exponential formula does not take into account the discontinuity of tracks and thus overestimated the radiation damage.

We can conclude that there is no conflicting effect of increasing $J_{c}$ due to a larger number of pinning centers and decreasing $J_{c}$ due to the reduced superconducting volume fraction arising from columnar continuous tracks, besides our damaged fractions are much lower than those shown in $[10,11]$. Therefore, the critical current density measured in our experiments $3 \mathrm{MA} \cdot \mathrm{cm}^{-2}$ at $77 \mathrm{~K}(0$ $\mathrm{T}, 46 \mathrm{MeV} \mathrm{Xe}$ ions) is one order of magnitude higher than obtained in $[10,11]$.

Numerous small damaged regions - the aligned in the ion beam direction spheroids from discontinues tracks - with sizes around $\xi^{3}$ ( $\xi$ in ab plane is the coherence length ranged from $5 \mathrm{~nm}$ to $2 \mathrm{~nm}$ between $77 \mathrm{~K}$ to $10 \mathrm{~K}$ respectively [30]) usually considered as weak pinning centers. 
However, they become an increasing element to pin magnetic flux lines providing the high current density due to the large pinning force (Fig. 12) at low $(4.2-35 \mathrm{~K})$ temperatures in the moderate magnetic fields compared to the continuous tracks.

The sample irradiated with $77 \mathrm{MeV}$ Xe ions didn't bring the expected pinning effect and critical current increase though it contains discontinuous tracks with spheroids and ellipsoids, but which number is less and size larger than in sample irradiated with $46 \mathrm{MeV} \mathrm{Xe}$ ions. Therefore, a mixture of such pinning centers occupies an intermediate position between strong large pinning centers (continuous tracks) and numerous weak small pinning centers and - how it was mentioned in [31] - this behavior supports the assumption that different structural defects complements each other in a mixed-pinning landscape. Nevertheless, in opposite to the absolute values of $J_{c}$ measured in the $77 \mathrm{MeV}$ Xe irradiate sample, its lift-factors better highlight the improvement of conducting properties in magnetic fields compared to the reference. Thus, our measurements showed the benefit of the use of the least energetic ions among energies considered in this work (about 0.13 $\mathrm{MeV} /$ nucleon).

\section{CONCLUSIONS}

We have studied the effect of irradiation with Xe ions of different energy in the range from 0.13 to $0.76 \mathrm{Mev} /$ nucleon on the microstructure and critical current density of YBCO based 2G HTS wires under magnetic fields up to $8 \mathrm{~T}$ and temperatures from 4.2 to $77 \mathrm{~K}$.

Continuous or discontinuous tracks with disordered (amorphous) structure produced by Xe ions with primary energies 167,77 and $46 \mathrm{MeV}$ were observed in a $1 \mu \mathrm{m}$ thick YBCO layer under a $2 \mu \mathrm{m}$ thick Ag cap. HRTEM showed that only solidification of track material occurs. Quantitative EDXS evidenced that amount of all elements inside tracks is reduced compared with the composition of the matrix crystal and the material density of the continuous tracks drops from 6300 $\mathrm{kg} \cdot \mathrm{m}^{-3}$ for YBCO to $3600 \mathrm{~kg} \cdot \mathrm{m}^{-3}$. 
The reduction in superconducting volume corresponding to the disordered structure does not exceed $4 \%$ from the total sample volume. The tracks act as pinning centers for the magnetic flux lines. An enhancement of the critical current density is observed for every ion energy.

The largest critical current density occurs in the samples irradiated with the lowest one that corresponds to the highest number of pinning centers (spheroidal defects) along discontinuous tracks. These numerous pinning centers increase the $J_{c}$ in the low and intermediate magnetic fields $-56 \mathrm{MA} \mathrm{cm}^{-2}(0 \mathrm{~T}, 46 \mathrm{MeV} \mathrm{Xe}$ ions $)$ and $16 \mathrm{MA} \mathrm{cm}^{-2}(8 \mathrm{~T}, 46 \mathrm{MeV} \mathrm{Xe}$ ions $)$ at $4.2 \mathrm{~K} ; 3 \mathrm{MA} \mathrm{cm}^{-2}$ (0 T, $46 \mathrm{MeV}$ Xe ions) and $1.6 \mathrm{MA} \mathrm{cm}^{-2}(8 \mathrm{~T}, 46 \mathrm{MeV}$ Xe ions) at $77 \mathrm{~K}$. If uncoated YBCO wire was considered, the energy of ions could be reduced to a level as low as $17.4 \mathrm{MeV}$.

\section{ACKNOWLEDGMENTS}

This work was supported by the Ministry of Science and Higher Education of the Russian Federation. 


\section{Figure captions}

FIG.1. Irradiation of the $2 \mathrm{G}$ HTS coated conductor wire with 46,77 and $167 \mathrm{MeV}$ Xe ions at the surface of Ag coating. The primary energies of Xe ions after passing the Ag layer reduced to $17.4,34.4$ and $101 \mathrm{MeV}$ at the $\mathrm{Ag} / \mathrm{YBCO}$ interface.

FIG. 2. Continuous (and nearly continuous) tracks produced by $167 \mathrm{MeV}$ Xe ions. The magnification of TEM images increases from (a) to (c); black-and-white arrows point forward at ion tracks and simple white arrows show rows of yttria precipitates (a). The orientation of the cross-section is close to the [100] YBCO and the direction of Xe ions is parallel to the [001] axis.

FIG. 3. Discontinuous tracks with spheroidal and prolate ellipsoidal radiation defects left by $77 \mathrm{MeV}$ Xe ions. The magnification of TEM images increases from (a) to (c); black-and-white arrows indicate the ion tracks and the simple white arrow show yttria precipitates.

FIG. 4. Discontinuous tracks with spheroidal radiation defects left by $46 \mathrm{MeV}$ Xe ions. The magnification of TEM images increases from (a) to (c); black-and-white arrows indicate the ion tracks and simple black arrows show yttria precipitates.

FIG. 5. TEM image of circular damaged regions (tracks) on plan-view samples (a, c, e) and corresponding SAED patterns (b, d, f) obtained along the [001] YBCO direction from the sample irradiated with $167 \mathrm{MeV}$ Xe ions (a, b), along the [101] YBCO direction from the sample-irradiated with $77 \mathrm{MeV}$ Xe ions (c, d) and along the [001] YBCO direction from the sample-irradiated with 46 $\mathrm{MeV}$ Xe ions; HAADF STEM image (g) and electron nanodiffractions from the pristine YBCO area (h) and from the ion track (i) along the [001] YBCO direction.

FIG. 6. HRTEM image (a) and the FFT (b) obtained along the [001] YBCO direction (arrows in FFT point the spot indices); insets around the radiation defect denoted as (1), (2), (3), (4), (5) are the HRTEM image simulation made for the defocus value of $33 \mathrm{~nm}$ and assumed thicknesses of $3.51 \mathrm{~nm}$ (1), $5.85 \mathrm{~nm}(2), 3.51 \mathrm{~nm}(3), 5.85 \mathrm{~nm}(4), 5.85 \mathrm{~nm}$ (5). Sample was irradiated with $46 \mathrm{MeV} \mathrm{Xe}$ ions. 
FIG.7. HRTEM image of YBCO matrix with radiation damaged regions (a), FFT (b) and the enlarge HRTEM image of the defect (c) obtained along the [101] YBCO direction; the insets in the enlarge HRTEM image denoted as (1), (2), (3), (4) are of the HRTEM image simulation performed for the defocus value of $66 \mathrm{~nm}$ and assumed thicknesses of $7.39 \mathrm{~nm}(1), 12.32 \mathrm{~nm}$ and tilt out of [101] zone axis $0.28^{\circ}(2), 12.32 \mathrm{~nm}$ and tilt $0.66^{\circ}(3), 13.32 \mathrm{~nm}$ and tilt $0.9^{\circ}$ (4). Sample was irradiated with $77 \mathrm{MeV}$ Xe ions.

FIG.8. HRTEM image (a), FFT (b) obtained along the [001] YBCO direction, enlarged HRTEM image (c) of the defect (arrow) and the HRTEM image simulation as insets denoted as (1), (2), (3) performed for the underdefocus value of $63 \mathrm{~nm}$ and assumed thicknesses of $8.18 \mathrm{~nm}$ and tilt $0.91^{\circ}$ (1), $10.52 \mathrm{~nm}$ and tilt $0.90^{\circ}(2), 10.52 \mathrm{~nm}$ and tilt $0.64^{\circ}(3), 13.32 \mathrm{~nm}$ and tilt $0.9^{\circ}$ (4). Sample was irradiated with $167 \mathrm{MeV}$ Xe ions.

FIG. 9. HAADF STEM image (a) and drift corrected EEL spectrum profile across the track produced by $167 \mathrm{MeV}$ Xe ions with points 1,2,3,4 (b) and points 4,5,6,7 (c). The area selected by the square is a reference for sample drift correction.

FIG. 10. HAADF STEM image (a) and drift corrected EEL spectrum profile across the track produced by $46 \mathrm{MeV}$ Xe ions with points 1, 2 (b) and points 3, 4 (c). The area selected by the square is a reference for sample drift correction.

FIG. 11. HAADF STEM image (a), distribution of $\mathrm{Y}, \mathrm{Ba}, \mathrm{Cu}$ elements in the EDXS map, (b), concentration profiles in net counts $(1,2$ and 3,4$)$ along the line scans across damaged regions pointed by arrows. Sample was irradiated with $167 \mathrm{MeV}$ Xe ions.

FIG. 12. Critical current density $J_{c}$ values versus magnetic field $B$ at temperatures $4.2,20,35,50$, 65 and $77 \mathrm{~K}$ for the reference sample (a) and samples irradiated with $46 \mathrm{MeV} \mathrm{Xe}$ ions and $5 \cdot 10^{11} \mathrm{~cm}^{-2}$ fluence (b), $77 \mathrm{MeV}$ Xe ions and $5 \cdot 10^{11} \mathrm{~cm}^{-2}$ fluence (c) and $167 \mathrm{MeV} \mathrm{Xe}$ ions and $1 \cdot 10^{11} \mathrm{~cm}^{-2}$ fluence. The error of measurements is $3-5 \%$.

FIG. 13. The difference on $J_{c}$ enhancement in sample irradiated with $46 \mathrm{MeV}$ Xe compared to 167 $\mathrm{MeV}$ Xe becomes important at large applied magnetic field. 
FIG. 14. Field dependence of pining force for the reference sample (a), for samples irradiated with Xe ions of the following energies and fluences: $46 \mathrm{MeV}$ and $5 \cdot 10^{11} \mathrm{~cm}^{-2}(\mathrm{~b}), 77 \mathrm{MeV}$ and $5 \cdot 10^{11}$ $\mathrm{cm}^{-2}(\mathrm{c}), 167 \mathrm{MeV}$ and $1 \cdot 10^{11} \mathrm{~cm}^{-2}(\mathrm{~d})$.

FIG. 15. Lift-factors versus Xe ion energy with fluencies $1 \cdot 10^{11}$ and $5 \cdot 10^{11} \mathrm{~cm}^{-2}$ calculated for magnetic fields in the range of $1 \mathrm{~T}$ and $8 \mathrm{~T}$ at temperature 77 (a) and $4.2 \mathrm{~K}(\mathrm{~b})$. The true energies of Xe ions entering the YBCO surface are displayed in the plots. 


\section{References}

${ }^{1}$ H. Watanabe, B. Kabius, K. Urban, B. Roas, S. Klaumtinzer, and G. Saemann-Ischenko, The influence of $173 \mathrm{MeV}$ Xe-ion irradiation on the microstructure of $\mathrm{YBa}_{2} \mathrm{Cu}_{3} \mathrm{O}_{7}$ thin films, Physica $\mathrm{C}$ 179, 75 (1991).

${ }^{2}$ D. Bourgault, M. Hervieu, S. Bouffard, D. Groult, and B. Raveau, 3.5 GeV Xe ion irradiation effects in the superconducting oxide $\mathrm{YBa}_{2} \mathrm{Cu}_{3} \mathrm{O}_{7-\delta}(\delta \leq 0.1)$ : A HREM investigation, Nucl. Instr. Methods. Phys. Res. 42, 61 (1989).

${ }^{3}$ Y. Yan, R.A. Doyle, A.M. Campbell, G. Wirth, and W.M. Stobbs, Modulated structure and local oxygen reordering induced by high-energy Au-ion irradiation in $\mathrm{YBa}_{2} \mathrm{Cu}_{3} \mathrm{O}_{7-\delta}$, Philos. Mag. Lett. 73, 299 (1996).

${ }^{4}$ L. A. Bursill and G. Braunshausen, Heavy-ion irradiation tracks in zircon, Philos. Mag. A 62, 395 (1990).

${ }^{5}$ W. Jiang, R. Devanathan, C.J. Sundgren, M. Ishimaru, K. Sato, T. Varga, S. Manandhar, and A. Benyagou, Ion tracks and microstructures in barium titanate irradiated with swift heavy ions: A combined experimental and computational study, Acta Mater. 61, 7904 (2013)

${ }^{6}$ J.-H. Kwon, Y. Meng, L. Wu, Y. Zhu, Y. Zhang, V. Selvamanickam, U. Welp, W.-K. Kwok, and J.-M. Zuo, Extended electronic structure inhomogeneity created by double chain layer defect surrounding columnar tracks in heavy-ion irradiated $\mathrm{YBa}_{2} \mathrm{Cu}_{3} \mathrm{O}_{7-\delta}$, Supercond. Sci. Technol. 31, 105006 (2018)

${ }^{7}$ E.I. Suvorova, M. Cantoni, P.A. Buffat, A.Y. Didyk, L.K. Antonova, A.V. Troitskii, and G.N. Mikhailova, Structure analysis of the YBCO layer in Ag/YBCO/metal oxide buffer/Hastelloy composite tape before and after $107 \mathrm{MeV} \mathrm{Kr}^{17+}$ irradiation, Acta Mater. 75, 71 (2014).

${ }^{8}$ T. A. Tombrello, Columnar track damage in YBCO, Nucl. Instr. Methods. Sect. B 95, 232 (1995). ${ }^{9}$ A.D. Marwick, L. Citiale, L. Krusin-Elbaum, R. Wheeler, J. R. Thompson, T.K. Worthington, M. A. Kirk, Y.R. Sun, H.R. Kerchner, and F. Holtzberg, Processing high-T superconductors with GeV heavy ions, Nucl. Instrum. Methods Phys. Res. Sect. B 80/81,1143 (1993) 
${ }^{10}$ R. Weinstein, R.-P. Sawh, A. Gandini, B. Mayes, and D. Parks, Is it really possible to increase $J_{c}$ by reducing the pinning potential? Supercond. Sci. Technol. 20, S167 (2007).

${ }^{11}$ R. Weinstein, R.-P. Sawh, D. Parks, and B. Mayes, Improvement of high $T_{c}$ superconductor by near-optimum pinning centers created by high Z, high-energy ions, Nucl. Instrum. Methods Phys. Res. Sect. B 272, 284 (2012).

${ }^{12}$ M. Leroux, K. J. Kihlstrom, S. Holleis, M. W. Rupich, S. Sathyamurthy, S. Fleshler, H. P. Sheng, D. J. Miller, S. Eley, L. Civale, A. Kayani, P. M. Niraula, U. Welp, and W.-K. Kwok, Rapid doubling of the critical current of $\mathrm{YBa}_{2} \mathrm{Cu}_{3} \mathrm{O}_{7-\delta}$ coated conductors for viable high-speed industrial processing. Appl. Phys. Lett. 107, 192601 (2015).

${ }^{13}$ M.W. Rupich, S. Sathyamurthy, S.Fleshler, Q. Li, V. Solovyov, T. Ozaki, U. Welp, W.-K. Kwok, M. Leroux, A.E. Koshelev, D.J. Miller, K. Kihlstrom, L. Civale, S. Eley, and A. Kayani, Engineered Pinning Landscapes for Enhanced 2G Coil Wire. IEEE Trans. Appl. Supercond., 26, 6601904 (2016).

${ }^{14}$ Y. L. Wang, L. R. Thoutam, Z. L. Xiao, B. Shen, J. E. Pearson, R. Divan, L. E. Ocola, G. W. Crabtree, and W. K. Kwok, Enhancing superconducting critical current by randomness. Phys. Rev. B 93, 045111 (2016).

${ }^{15}$ E.I. Suvorova, O.V. Uvarov, A. V. Ovcharov, I.A. Karateev, A.L.Vasiliev, V.A. Skuratov, and P.A. Buffat, Effects of $\mathrm{Kr}$ and $\mathrm{Xe}$ ion irradiation on the structure of $\mathrm{Y}_{2} \mathrm{O}_{3}$ nanoprecipitates in $\mathrm{YBCO}$ thin film conductors, Philos. Mag. B 98, 3127 (2018).

${ }^{16}$ Y. Zhu, Z. X. Cai, R. C. Budhani, M. Suenaga, and D. O. Welch, Structures and effects of radiation damage in cuprate superconductors irradiated with several-hundred-MeV heavy ions, Phys. Rev. B 48, 6436 (1993).

${ }^{17}$ J. F. Ziegler and J. P. Biersack, SRIM (The stopping and range of ions in matter) software available at http://www.srim.org/SRIM/SRIMLEGL.htm (2013).

${ }^{18}$ P. Stadelmann JEMS: java electron microscopy software, http://www.jems-swiss.ch/, (2019). 
${ }^{19} \mathrm{M}$. Toulemonde, S. Bouffard, and F. Studer Swift heavy ions in insulating and conducting oxides: tracks and physical properties, Nucl. Instrum. Methods Phys. Res. Sect. B 91, 108 (1994).

${ }^{20}$ N. M. Strickland, E.F. Talantsev, N. J. Long, J. A. Xia, S. D. Searle, J. Kennedy, A. Markwitz, M. W. Rupich, X. Li, and S. Sathyamurthy. Flux pinning by discontinuous columnar defects in 74 MeV Ag-irradiated $\mathrm{YBa}_{2} \mathrm{Cu}_{3} \mathrm{O}_{7}$ coated conductors. Physica C 469, 2060 (2009).

${ }^{21}$ D. O. Shvedchenko and E. I. Suvorova, New Method of Automated Statistical Analysis of Polymer-Stabilized Metal Nanoparticles in Electron Microscopy Images, Crystallography Rep, 62, 802 (2017).

${ }^{22}$ D.C. Johnston, A.J. Jacobson, J.M. Newsam, J.T. Lewandowski, D.P. Goshorn, D. Xie, and W.B. Yelon, Variation in the structural, magnetic, and superconducting properties of $\mathrm{YBa}_{2} \mathrm{Cu}_{3} \mathrm{O}_{7-\mathrm{x}}$ with oxygen content. In: ACS symposium series, 351, p. 136 [Chapter 14] (1987).

${ }^{23}$ N. Nucker, H. Romberg, X. X. Xi, and J. Fink, Symmetry of holes in high-Tc superconductors, Phys. Rev. B 39, 6619 (1989).

${ }^{24}$ F. F. Komarov, and V. A. Belyi, Fluctuation mechanism of formation of discontinuous tracks by fast ions in solids, J. Exp Theor Phys+, 95, 316 (2002).

${ }^{25}$ F. F. Komarov, Defect and track formation in solids irradiated by superhigh-energy ions, Physics - Uspekhi 46, 1253 (2003).

${ }^{26}$ P.I. Gaiduk, F.F. Komarov, and W. Wesch, Damage evolution in crystalline InP during irradiation with swift Xe ions, Nucl. Instrum. Methods Phys. Res. Sect. B 164-165, 377 (2000).

27 P. Kluth, C. S. Schnohr, O. H. Pakarinen, F. Djurabekova, D. J. Sprouster, R. Giulian, M. C. Ridgway, A. P. Byrne, C. Trautmann, D. J. Cookson, K. Nordlund, and M. Toulemonde, Fine Structure in Swift Heavy Ion Tracks in Amorphous $\mathrm{SiO}_{2}$, Phys. Rev. Lett. 101, 175503 (2008).

${ }^{28}$ E.M. Gyorgy, R.B. J. K.A. van Dover, L.F. Schneemeyer, and J.V. Waszczak, Anisotropic critical currents in $\mathrm{YBa}_{2} \mathrm{Cu}_{3} \mathrm{O}_{7}$ analyzed using an extended Beam model, Appl. Phys. Lett. 55, 283 (1989). ${ }^{29}$ W-J. Oh, J.-E. Kim, and Sang-Im Yoo, Enhanced pinning properties of $\mathrm{GdBa}_{2} \mathrm{Cu}_{3} \mathrm{O}_{7-\delta}$ films with the $\mathrm{Gd}_{2} \mathrm{O}_{3}$ nanoparticles, IEEE Trans. On. Appl. Supercond. 27, 8000605 (2017). 
${ }^{30}$ J. P. F. Feighan, A. Kursumovic, and J. L. MacManus-Driscoll, Materials design for artificial pinning centres in superconductor PLD coated conductors, Supercond. Sci. Technol. 30, 123001 (2017).

${ }^{31}$ W.-K. Kwok, U. Welp, A.Glatz, A.E Koshelev, K. J. Kihlstrom, and G. W Crabtree. Vortices in high-performance high-temperature superconductors. Rep. Prog. Phys. 79, 116501 (2016). 
Scheme of irradiation

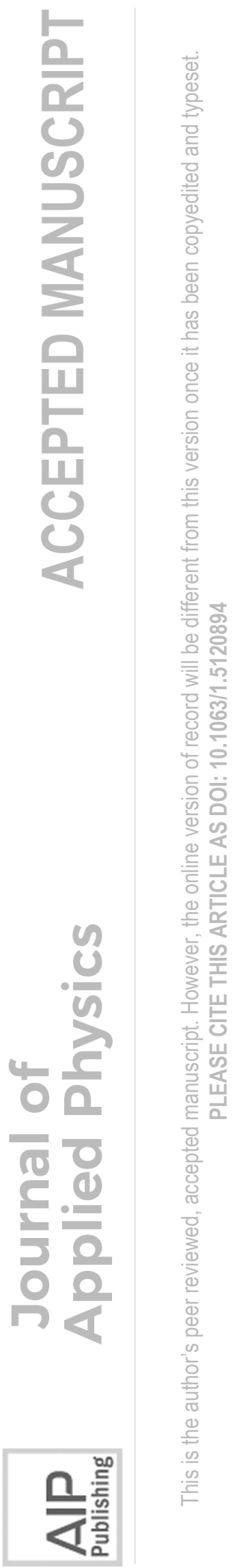

${ }^{132} \mathrm{Xe}$ ions

$46 \mathrm{MeV}$,

$77 \mathrm{MeV}$, $167 \mathrm{MeV}$

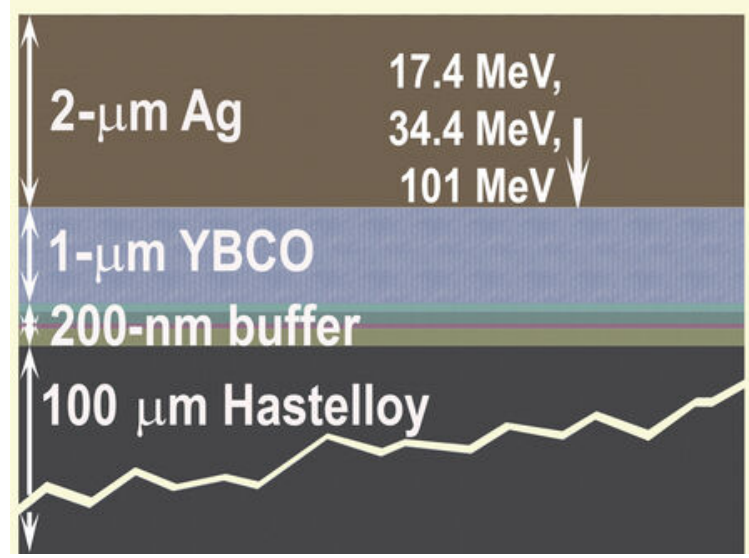



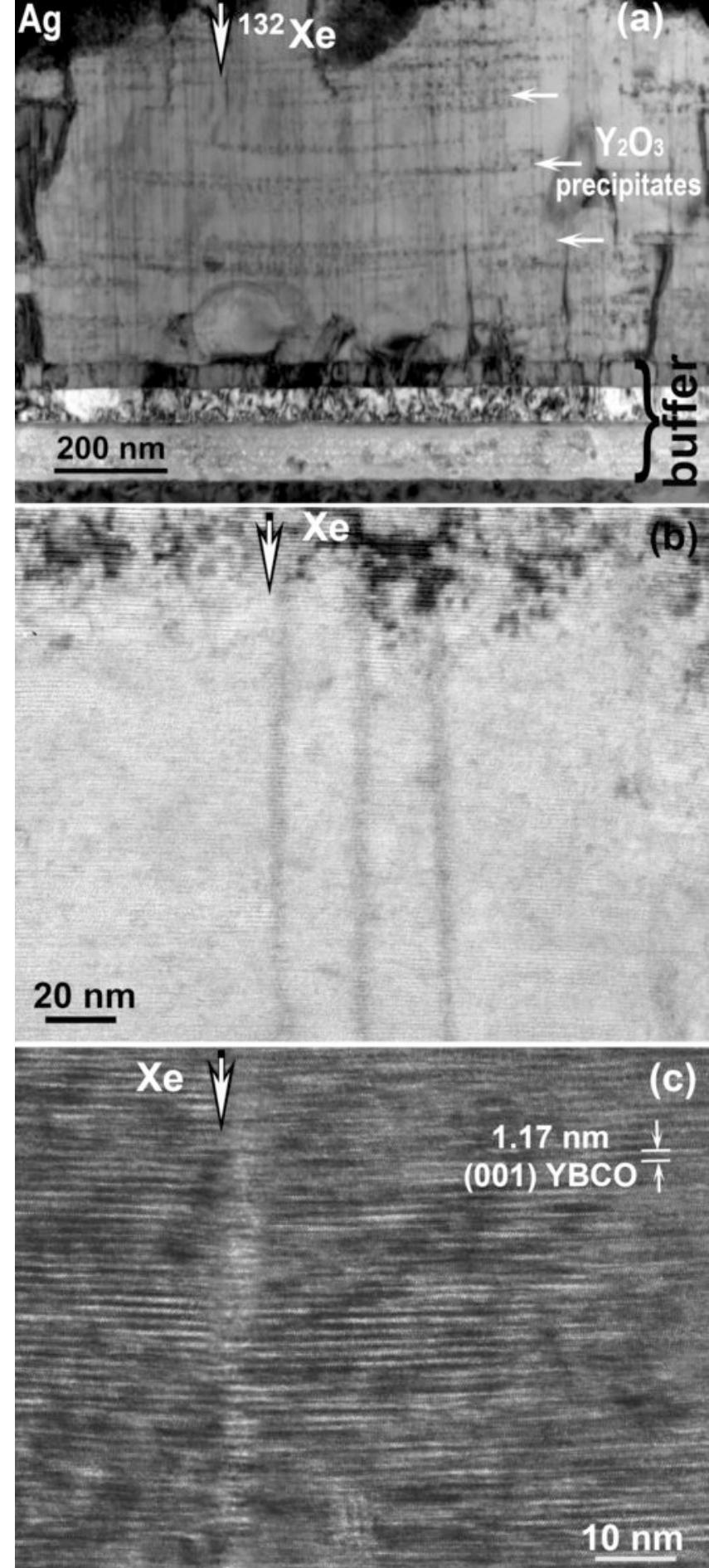

碞产 


\section{$100 \mathrm{~nm}$}
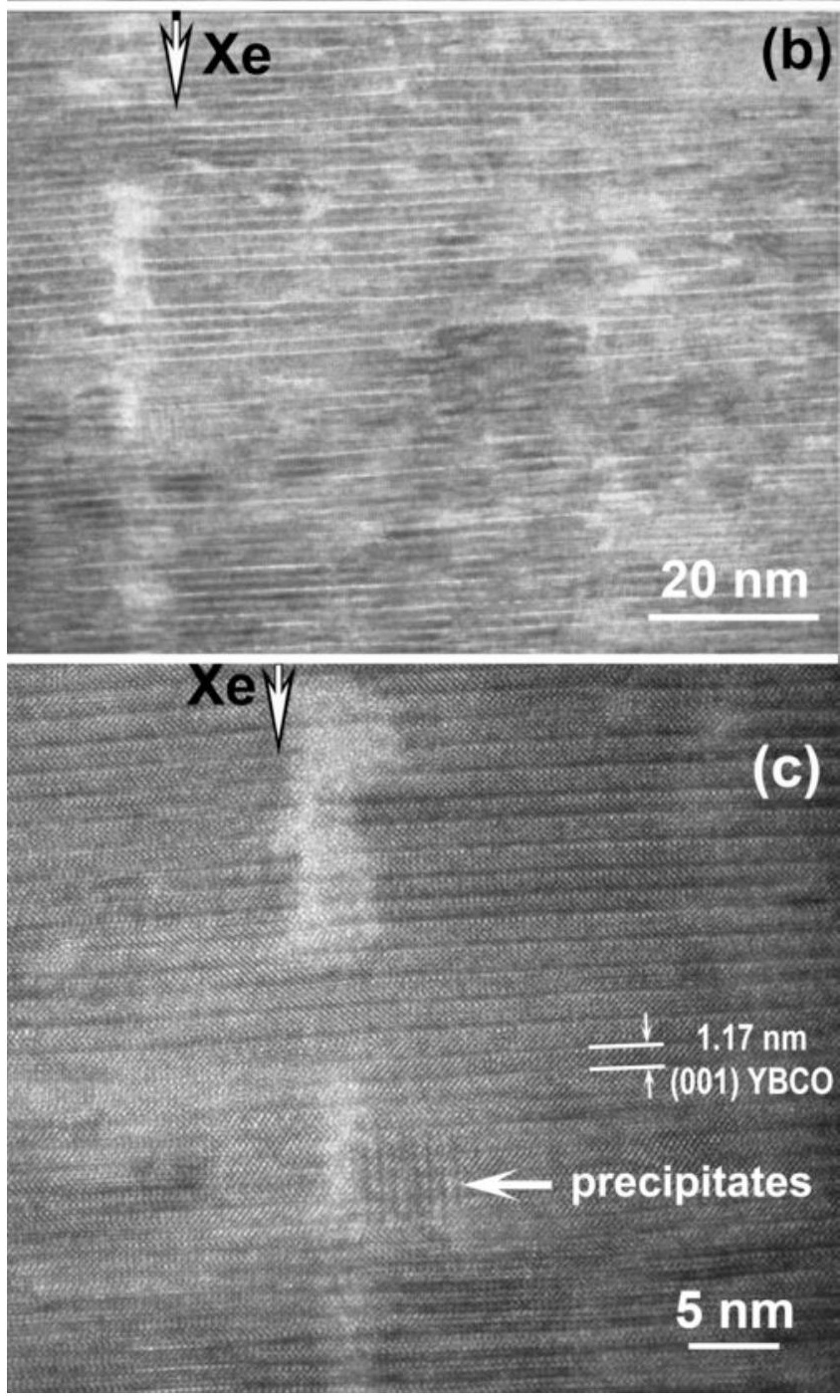

은 


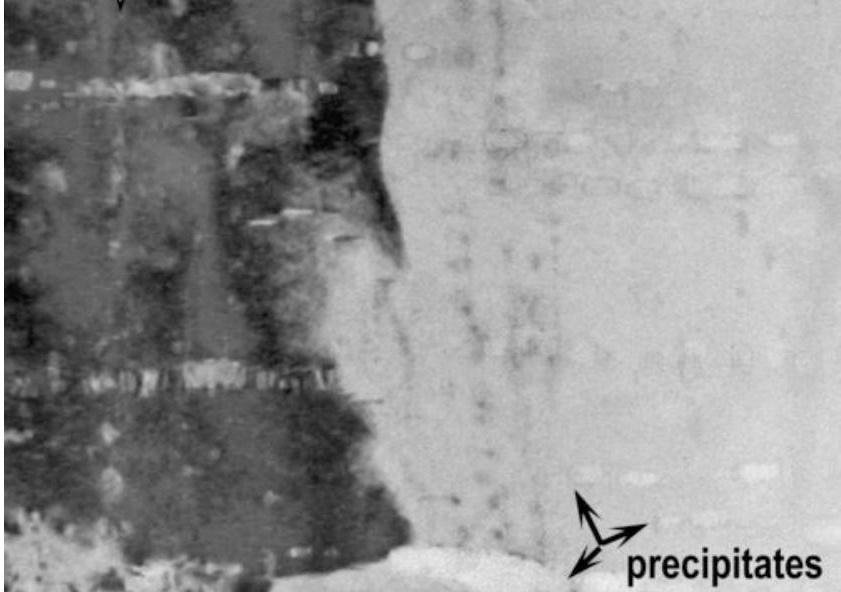

$50 \mathrm{~nm}$

precipitates

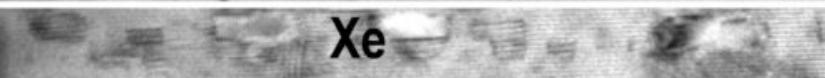
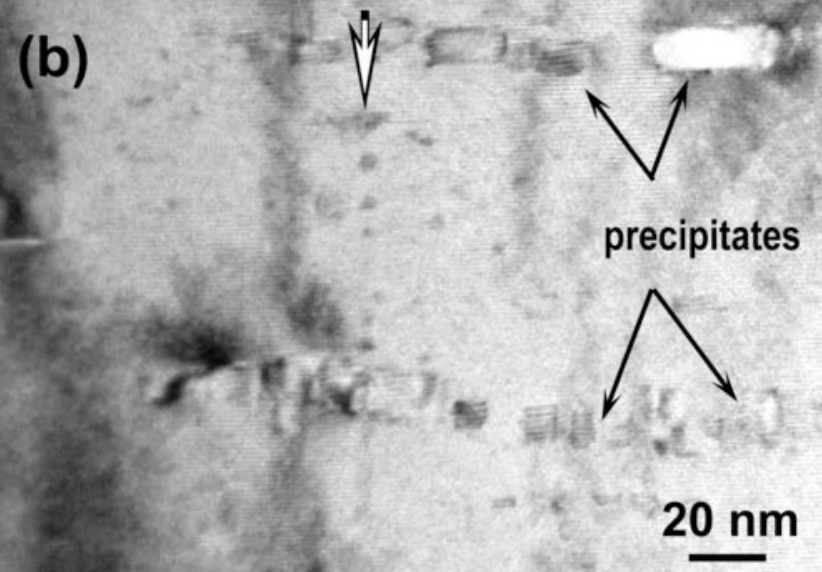

U

(U)

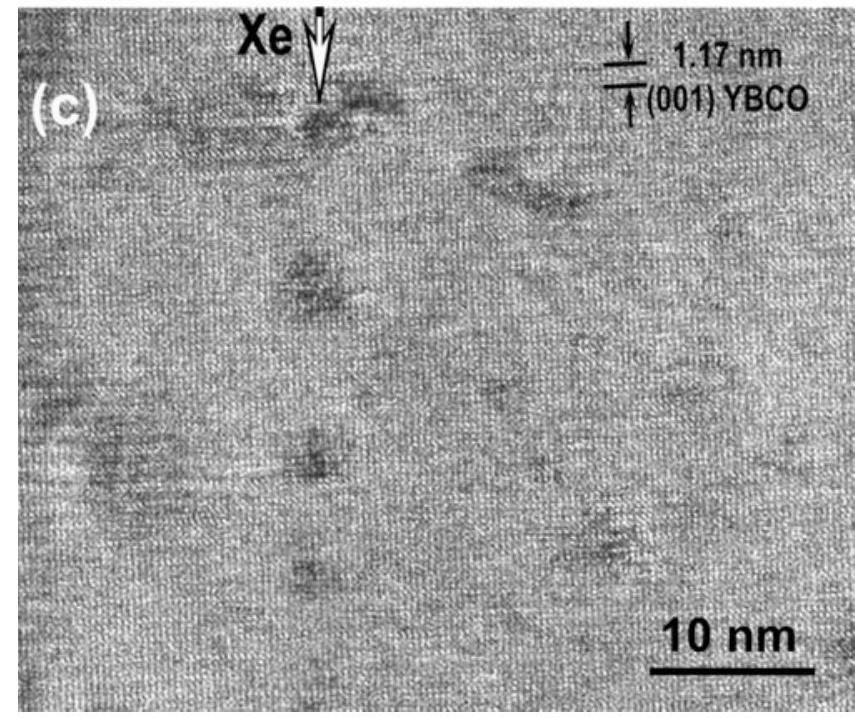

猔空

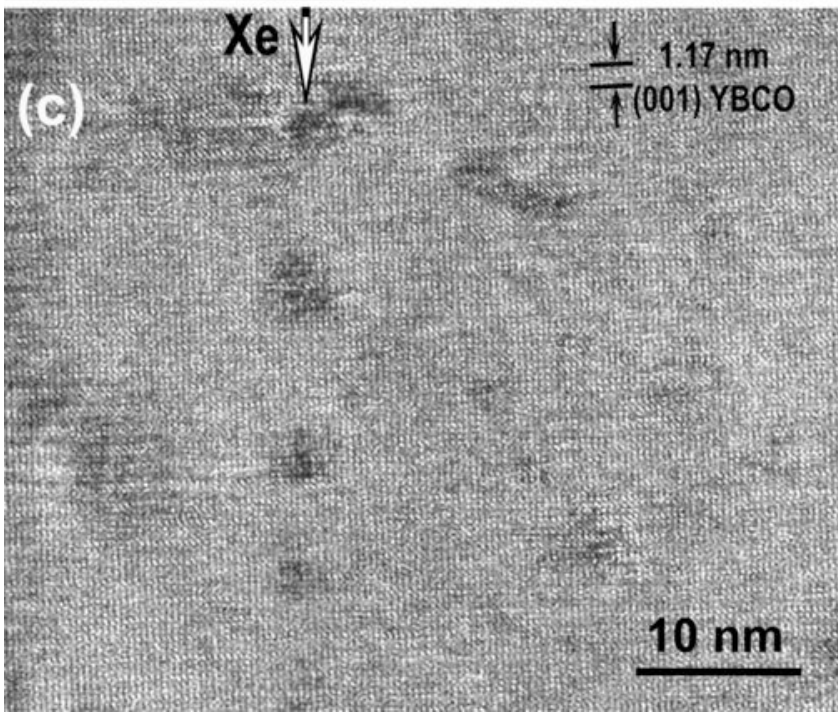

는 
(a)

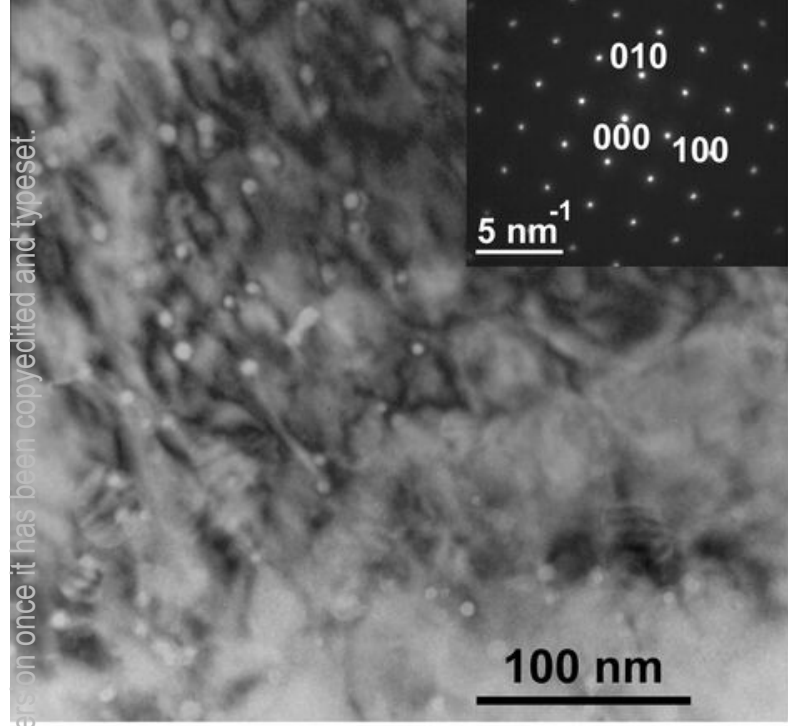

$\frac{1}{4}$

$\frac{1}{4}$

$\frac{1}{4}$
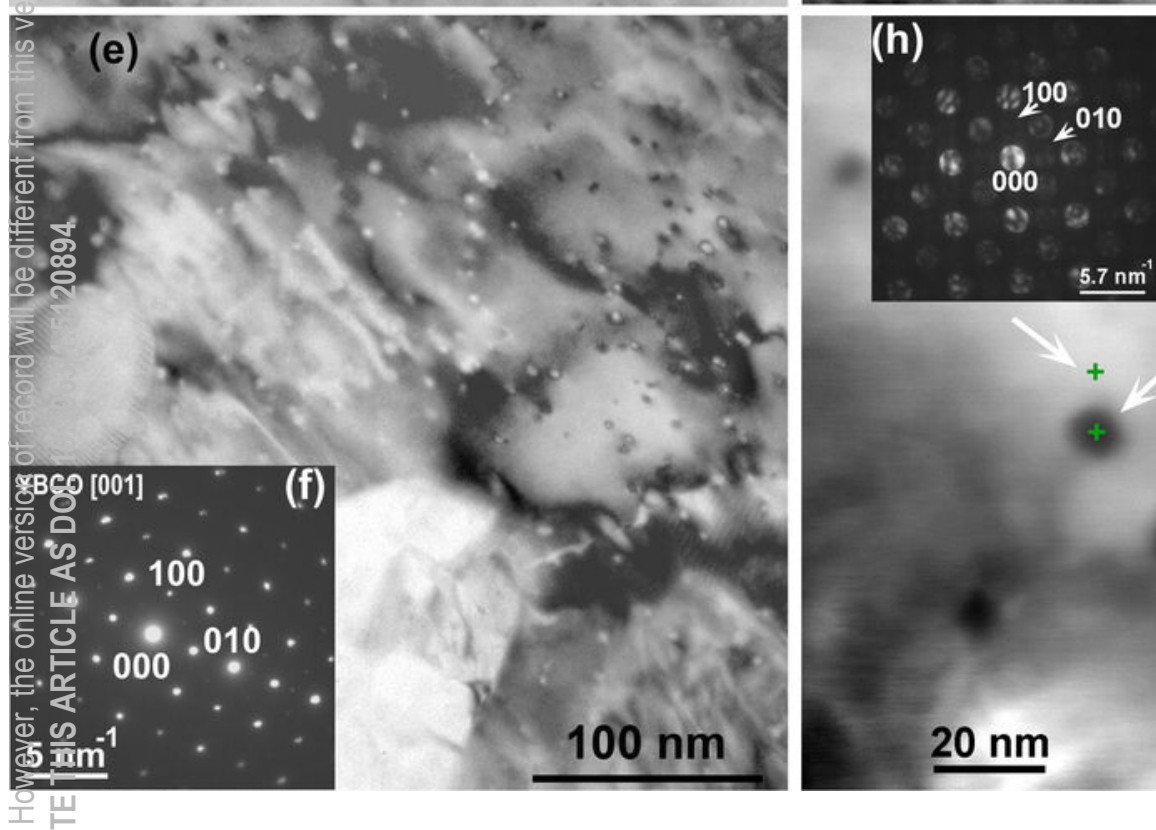

(c)

\section{$20 \mathrm{~nm}$}

$50 \mathrm{~nm}$

(d)

YBCO [101] *

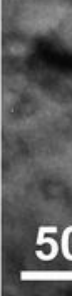

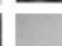

101.010

.000
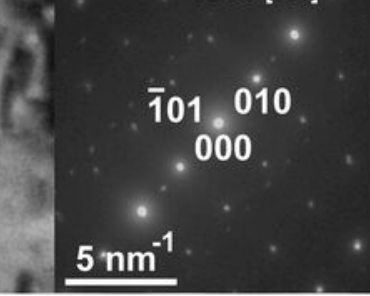

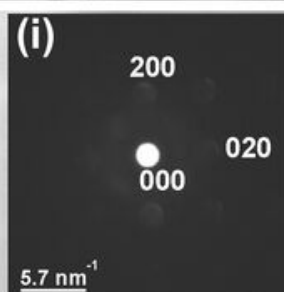

(g)

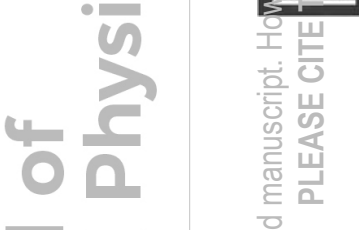

ro

는 


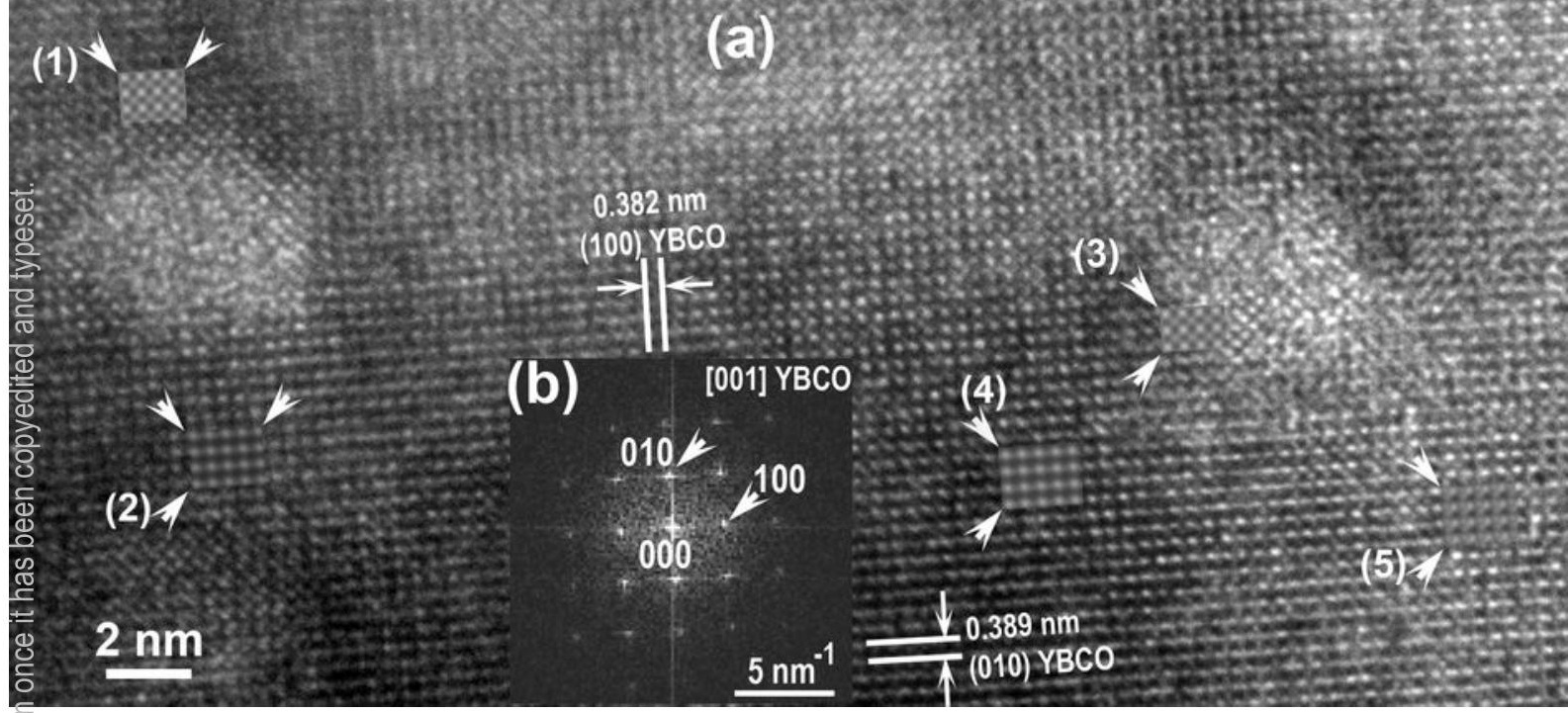

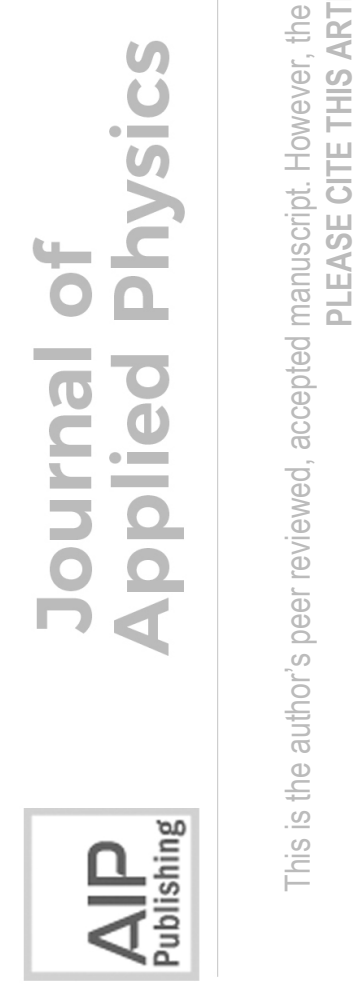




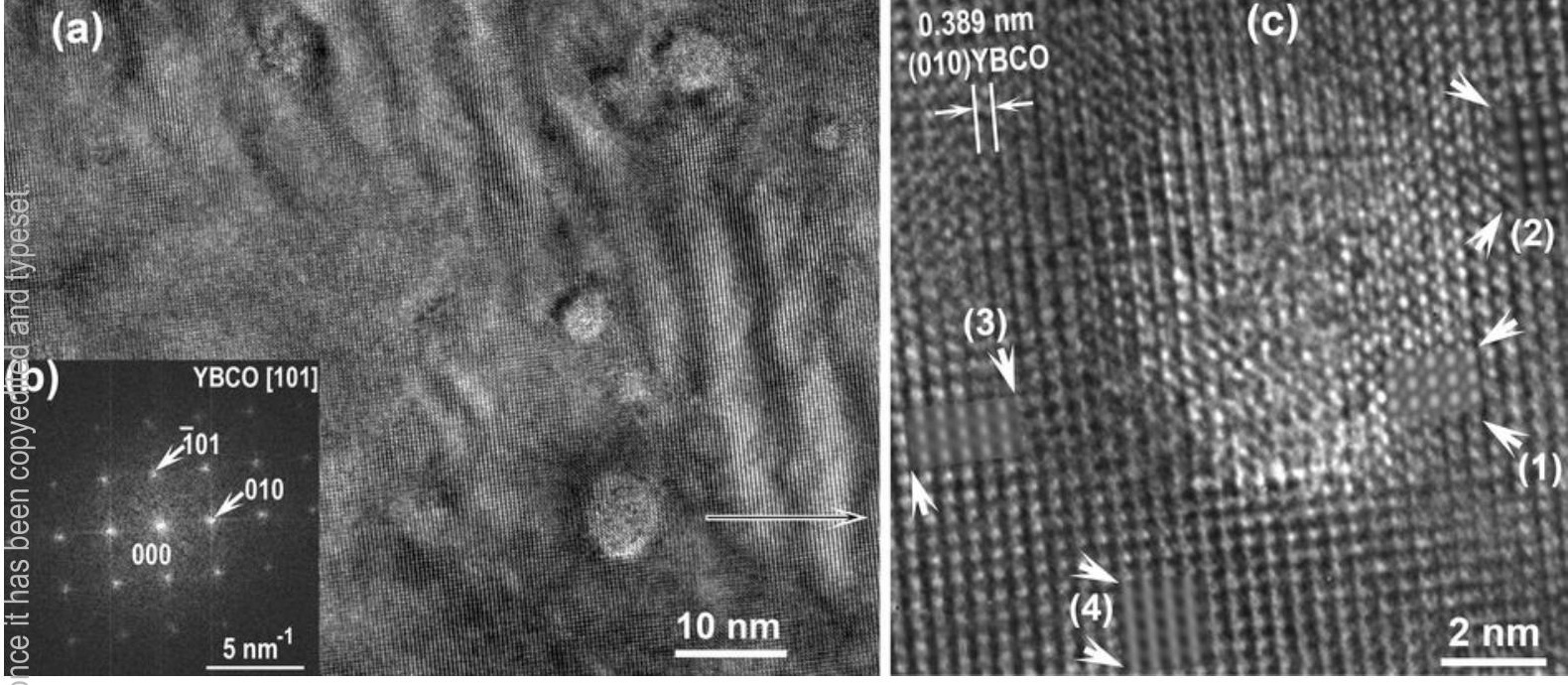

峁

음 


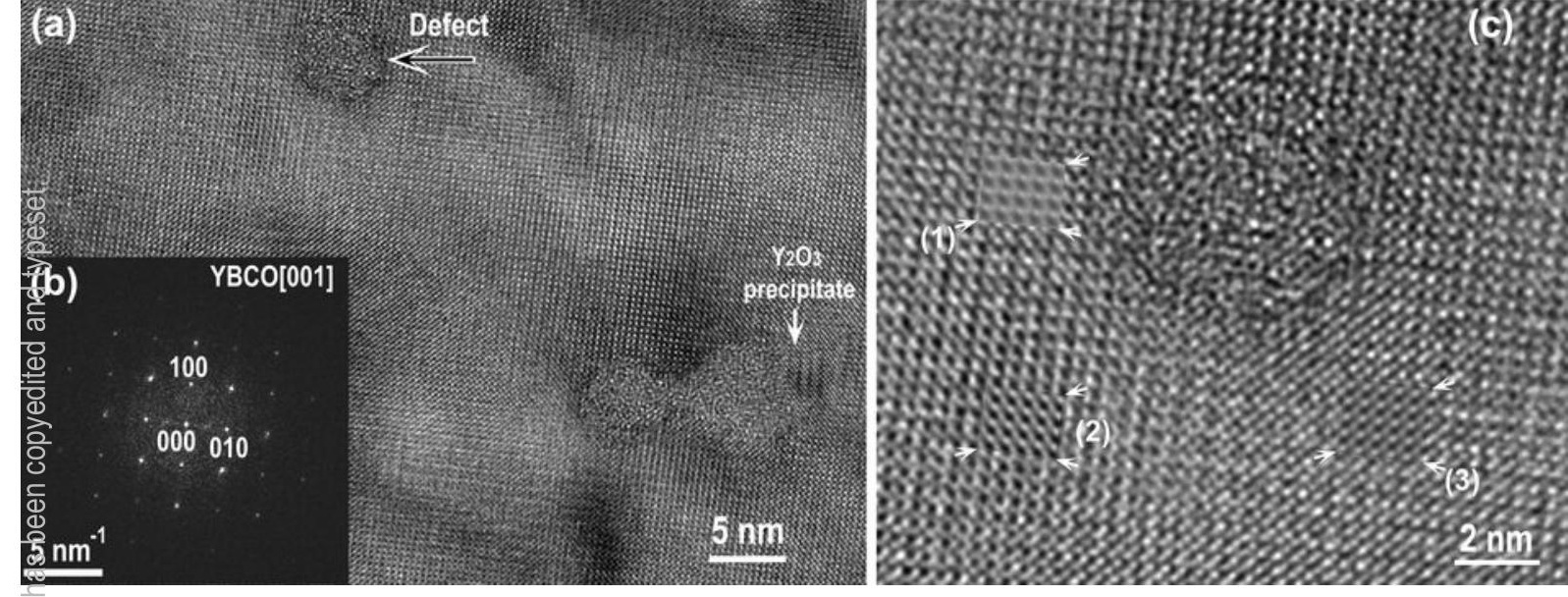



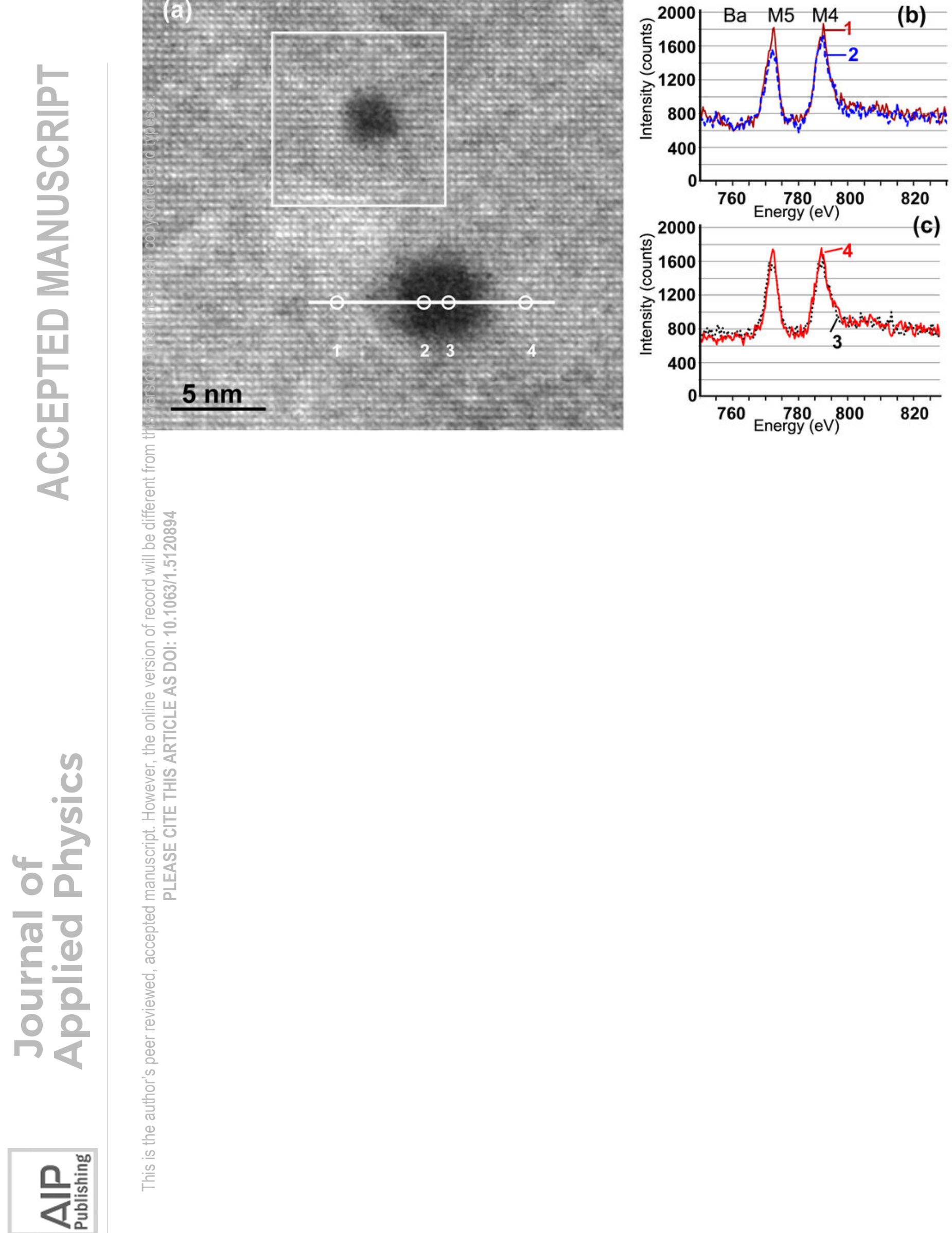

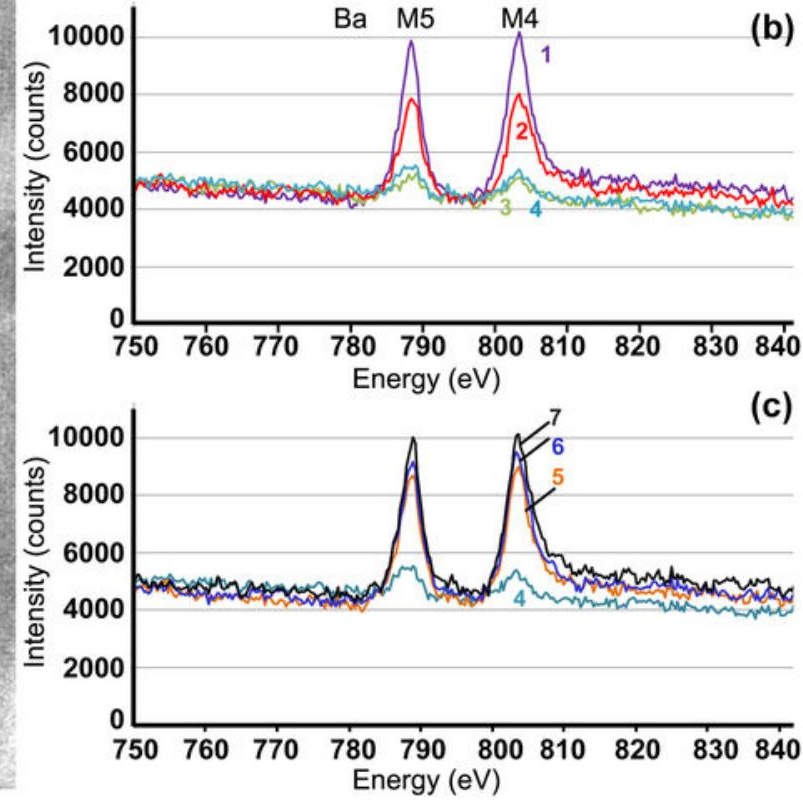

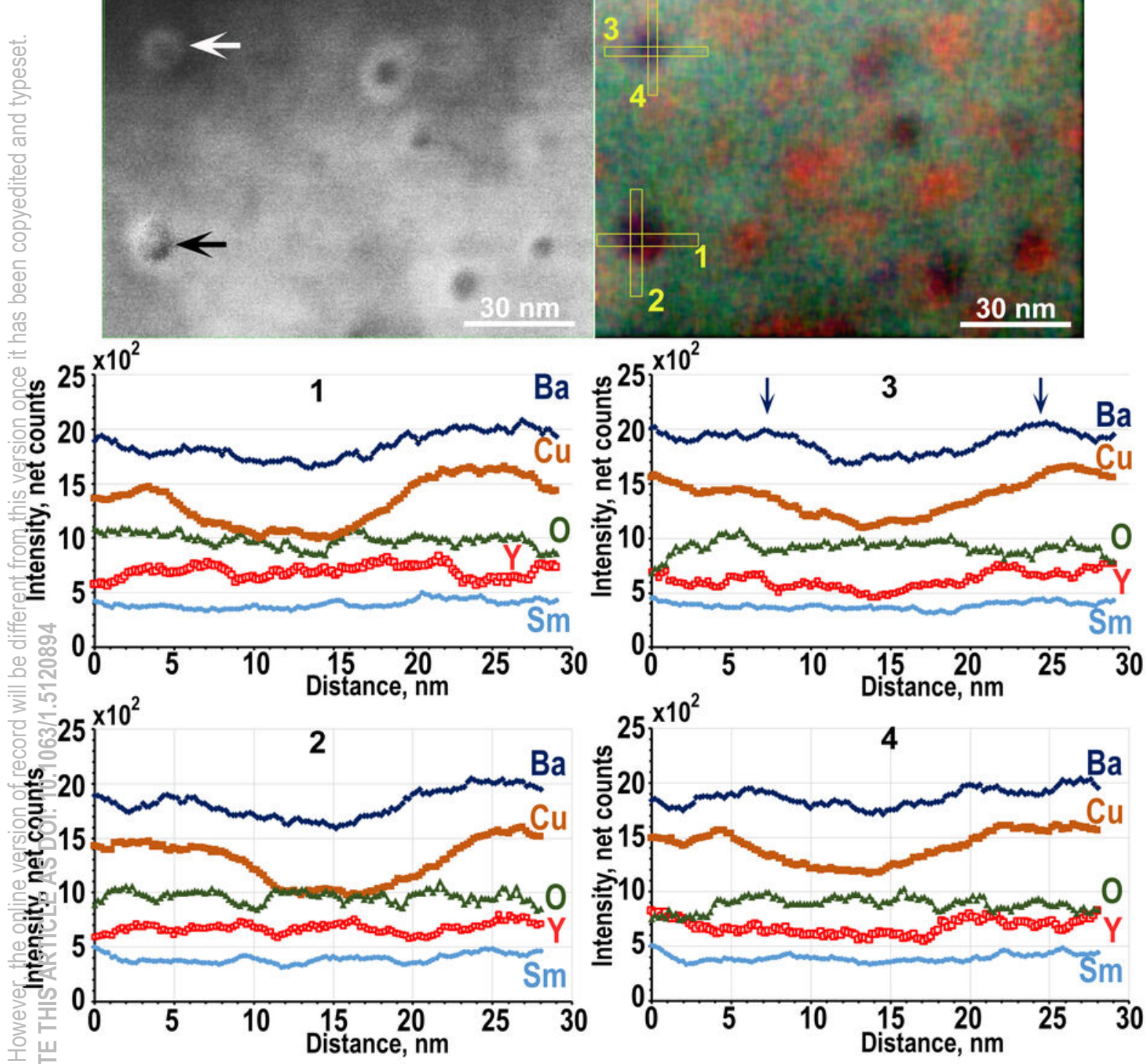

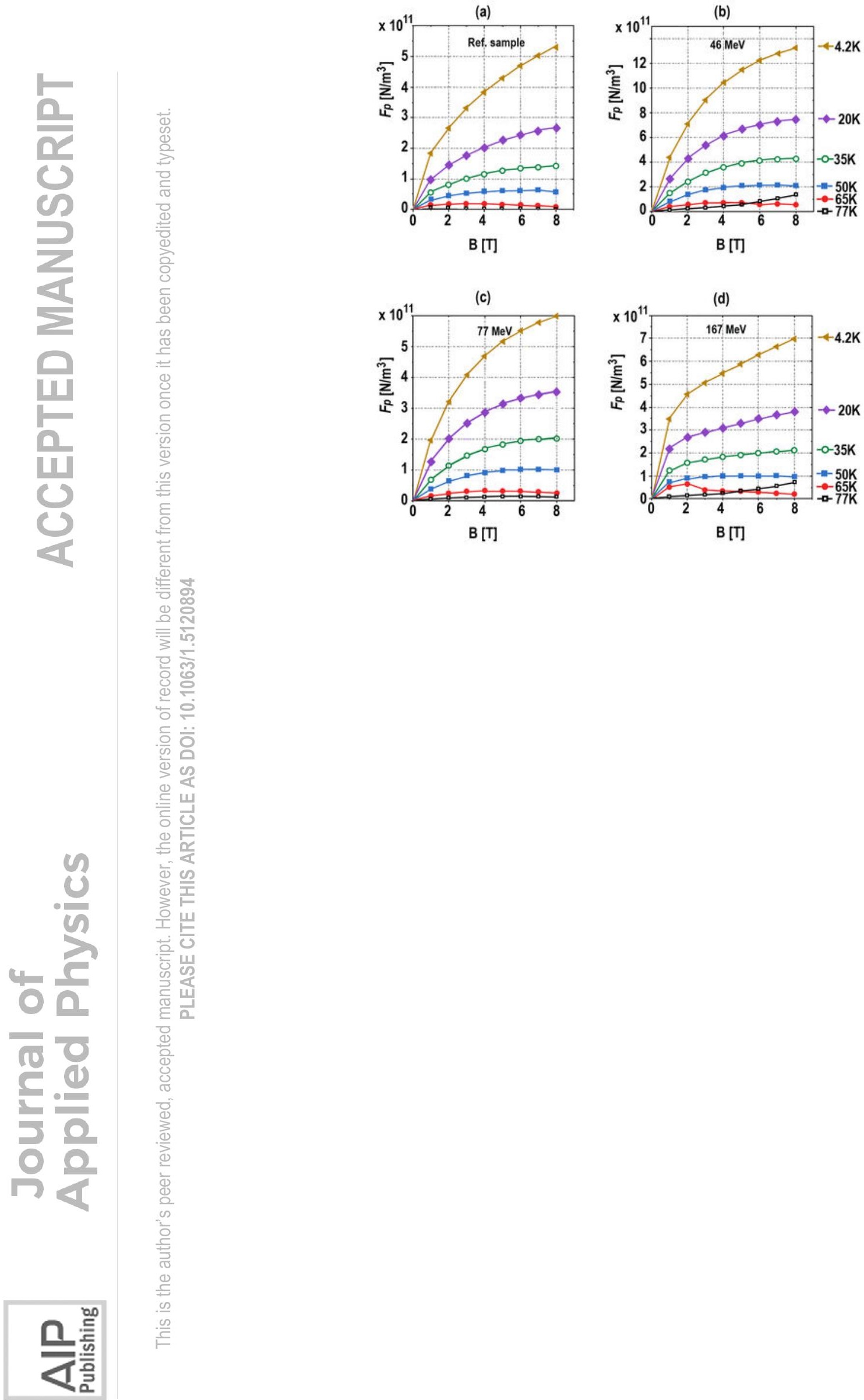
(a)

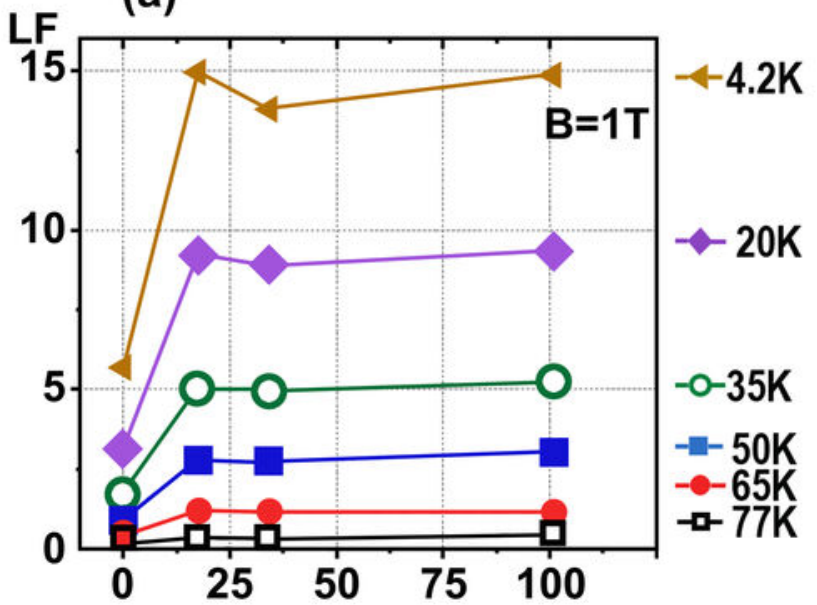

(b)

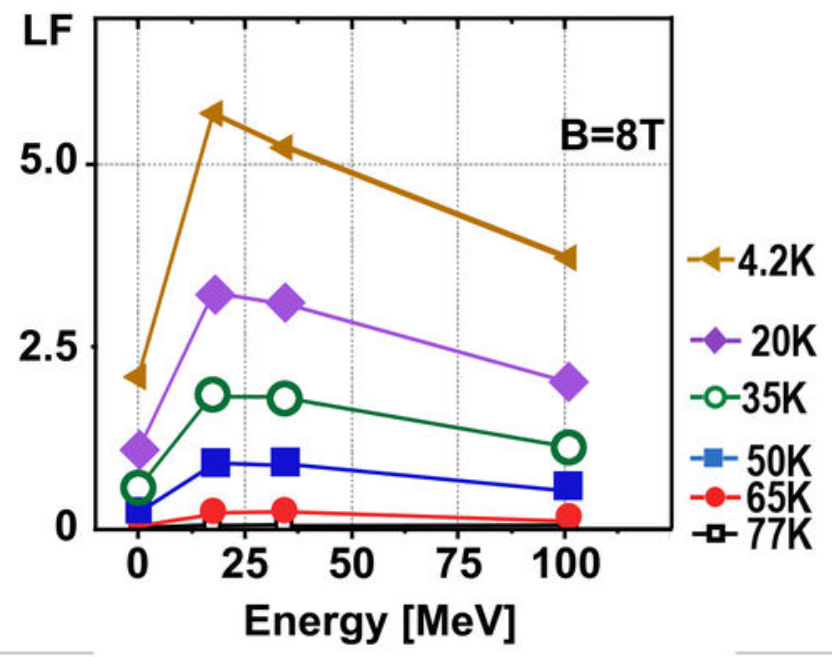

